\title{
On Identification of the Threshold Diffusion Processes
}

\author{
Yury A. Kutoyants \\ Laboratoire de Statistique et Processus, Université du Maine \\ 72085 Le Mans, Cédex 9, France
}

\begin{abstract}
We consider the problems of parameter estimation for several models of threshold ergodic diffusion processes in the asymptotics of large samples. These models are the direct continuous time analogues of the well-known in time series analysis threshold autoregressive (TAR) models. In such models the trend is switching when the observed process atteints some (unknown) values and the problem is to estimate it or to test some hypotheses concerning these values. The related statistical problems correspond to the singular estimation or testing, for example, the rate of convergence of estimators is $T$ and not $\sqrt{T}$ as in regular estimation problems. We study the asymptotic behavior of the maximum likelihood and bayesian estimators and discuss the possibility of the construction of the goodness of fit test for such models of observation.
\end{abstract}

Key words: parameter estimation, threshold models, singular estimation, ergodic diffusion process, goodness of fit test, Cramer-von Mises type tests. MSC 2000 Classification: 62M02, 62G10, 62G20.

\section{Introduction}

The simplest example of the threshold model is the following threshold autoregressive (TAR) time series:

$$
X_{j+1}=\varrho_{1} X_{j} \mathbb{I}_{\left\{X_{j}<\vartheta\right\}}+\varrho_{2} X_{j} \mathbb{I}_{\left\{X_{j} \geq \vartheta\right\}}+\varepsilon_{j+1}, \quad j=0, \ldots, n-1,
$$

where $\varepsilon_{j}$ are i.i.d. $\mathcal{N}\left(0, s^{2}\right), \varrho_{1} \neq \varrho_{2}$ and $\left|\varrho_{i}\right|<1$. Therefore we have two different autoregressive processes depending on the region of observations 
$\{x: x<\vartheta\}$ or $\{x: x \geq \vartheta\}$. This time series has ergodic properties with invariant density close to a weighted sum of two Gaussian densities. If we suppose that $s^{2}, \varrho_{1}, \varrho_{2}$ are known and $\vartheta \in \Theta=(\alpha, \beta)$ is unknown parameter, then we obtain the first problem of threshold $\vartheta$ estimation. It is easy to see that the likelihood ratio is a piece wise constant (discontinuous) function of $\vartheta$, the Fisher information is equal infinity. As usual in singular estimation problems, the rate of convergence of maximum likelihood $\hat{\vartheta}_{n}$ or Bayesian $\tilde{\vartheta}_{n}$ estimators is $n$ and not $\sqrt{n}$ i.e.; the quantities $n\left(\hat{\vartheta}_{n}-\vartheta\right)$ and $n\left(\tilde{\vartheta}_{n}-\vartheta\right)$ have non degenerate limits.

There are many different threshold regression models of such type extensively developed in econometrics and, of course, the identification of these models attracts attention of statisticians (see, e.g. the works by Quandt (1958), Tong (1990) [10], Chan (1993) [1], Hansen (2000) [6], Fan and Yao (2003) [5], Koul et al. [11, Chan and Kutoyants 2] and the references therein). Note that continuous time models actually find a wide range of applications in econometrical problems and occupy a central place in financial mathematics (see, e.g., the work by Shreve [20]).

Our goal is to study several models of continuous time analogues (diffusion processes) of such threshold type time series and to describe the properties of estimators of the thresholds for these models. Note that the general theory of parameter estimation (in regular case) for ergodic diffusion processes is actually well developped (see, e.g. [13], 21] and references therein) but the problems of threshold estimation are of singular type and need a special consideration. To illustrate these statements of the problem let us consider the following process

$$
\mathrm{d} X_{t}=-\rho_{1} X_{t} \mathbb{1}_{\left\{X_{t}<\vartheta\right\}} \mathrm{d} t-\rho_{2} X_{t} \mathbb{1}_{\left\{X_{t} \geq \vartheta\right\}} \mathrm{d} t+\sigma \mathrm{d} W_{t}, \quad 0 \leq t \leq T,
$$

where $W_{t}$ is Wiener process, $\rho_{1} \neq \rho_{2}$ and $\rho_{i}>0$. We call it Threshold Ornstein-Uhlenbeck (TOU) process because it can be considered as a mixture of two different Ornstein-Uhlenbeck processes with switching. If we suppose that $\sigma, \rho_{1}, \rho_{2}$ are known and $\vartheta \in \Theta=(\alpha, \beta)$ is unknown parameter then we obtain the problem of parameter (threshold) $\vartheta$ estimation.

It is in some sense similar to TAR (1) and the link between them can be clarified by the following consideration. Let us consider the discrete time approximation of the process (2) with $t_{j}=j \delta, j=1, \ldots, n-1$, where $\delta=$ $T / n$, then we obtain

$$
X_{t_{j+1}}=\left(1-\rho_{1} \delta\right) X_{t_{j}} \mathbb{I}_{\left\{X_{t_{j}}<\vartheta\right\}}+\left(1-\rho_{2} \delta\right) X_{t_{j}} \mathbb{I}_{\left\{X_{t_{j}} \geq \vartheta\right\}}+\sigma\left[W_{t_{j+1}}-W_{t_{j}}\right] .
$$

This process coincides with (11) if we put $X_{j}=X_{t_{j}}, \varrho_{i}=\left(1-\rho_{i} \delta\right)$ and $\varepsilon_{j+1}=\sigma\left[W_{t_{j+1}}-W_{t_{j}}\right] \sim \mathcal{N}\left(0, \sigma^{2} \delta\right)$, i.e., $s^{2}=\sigma^{2} \delta$. Hence, the regression model (1) is a discrete time approximation of the TOU process (2). 
The threshold estimation problems for both models are of singular type and the limit distributions of the MLE's $n\left(\hat{\vartheta}_{n}-\vartheta\right)$ and $T\left(\hat{\vartheta}_{T}-\vartheta\right)$ are of argsup type functionals of the compound Poisson and Wiener processes respectively.

The process $\left(X_{t}\right)_{t>0}$ has ergodic properties, the invariant density is a mixture of two Gaussian, the Fisher information is equal to infinity and we show that the maximum likelihood and Bayesian estimators converge to two different limit laws.

We consider several other threshold type models of ergodic diffusion processes and study the asymptotic properties of the ML and Bayesian estimators. We discuss as well the construction of the goodness of fit tests for such threshold models.

\section{Threshold Ornstein-Uhlenbeck Process}

\subsection{Threshold estimation}

We start with the TOU process

$$
\mathrm{d} X_{t}=-\rho_{1} X_{t} \mathbb{1}_{\left\{X_{t}<\vartheta\right\}} \mathrm{d} t-\rho_{2} X_{t} \mathbb{1}_{\left\{X_{t} \geq \vartheta\right\}} \mathrm{d} t+\sigma \mathrm{d} W_{t}, \quad X_{0}, \quad 0 \leq t \leq T,
$$

where we suppose that the following condition is fulfilled.

Condition $\mathcal{A}$. The constants $\rho_{1} \neq \rho_{2}, \rho_{i}>0$ and $\sigma^{2}>0$ are known and the parameter $\vartheta \in \Theta=(\alpha, \beta), \alpha>0$ is unknown. The initial value $X_{0}$ is independent on the Wiener process random variable.

The value $\vartheta=0$ is excluded because in the case $\vartheta=0$ there is no jump in the trend coefficient and the properties of estimators are quite different.

We consider the problem of estimation of the threshold $\vartheta$ by the continuous time observations $X^{T}=\left(X_{t}, 0 \leq t \leq T\right)$ and we are interested by the asymptotic behavior of estimators as $T \rightarrow \infty$.

Note that the conditions $\mathcal{E S}$ of the existence of solution and $\mathcal{R} \mathcal{P}$ of the ergodicity are fulfilled (see [13], Sections 1.1 and 1.2) and the process $\left(X_{t}\right)_{t \geq 0}$ has ergodic properties with the invariant density

$$
f(\vartheta, x)=p_{1}(x, \vartheta) e^{-\frac{\rho_{1}\left(x^{2}-\vartheta^{2}\right)}{\sigma^{2}}}+p_{2}(x, \vartheta) e^{-\frac{\rho_{2}\left(x^{2}-\vartheta^{2}\right)}{\sigma^{2}}} .
$$

Here $p_{1}(x, \vartheta)=G(\vartheta)^{-1} \mathbb{1}_{\{x<\vartheta\}}$, and $p_{2}(x, \vartheta)=G(\vartheta)^{-1} \mathbb{1}_{\{x \geq \vartheta\}}$ and $G(\vartheta)$ is the normalizing constant. To simplify the exposition we suppose that the random variable $X_{0}$ has the density function $f(\vartheta, x)$, hence the observed process is stationary.

We are interested by the asymptotic behavior of the maximum likelihood and Bayesian estimators of the parameter $\vartheta$, therefore we need the likelihood 
ratio function $L\left(\vartheta, X^{T}\right)$. This function can be written as (see [16])

$$
\begin{aligned}
& \ln L\left(\vartheta, X^{T}\right)=-\frac{\rho_{1}}{\sigma^{2}} \int_{0}^{T} X_{t} \mathbb{I}_{\left\{X_{t}<\vartheta\right\}} \mathrm{d} X_{t}-\frac{\rho_{2}}{\sigma^{2}} \int_{0}^{T} X_{t} \mathbb{I}_{\left\{X_{t} \geq \vartheta\right\}} \mathrm{d} X_{t} \\
& -\frac{\rho_{1}^{2}}{2 \sigma^{2}} \int_{0}^{T} X_{t}^{2} \mathbb{I}_{\left\{X_{t}<\vartheta\right\}} \mathrm{d} t-\frac{\rho_{2}^{2}}{2 \sigma^{2}} \int_{0}^{T} X_{t}^{2} \mathbb{I}_{\left\{X_{t} \geq \vartheta\right\}} \mathrm{d} t+\ln f\left(\vartheta, X_{0}\right) .
\end{aligned}
$$

The contribution of the term $\ln f\left(\vartheta, X_{0}\right)$ is asymptotically negligeable and we will always omitted it for simplicity of exposition (see the details in [13]).

The MLE $\hat{\vartheta}_{T}$ and BE (for quadratic loss function) $\tilde{\vartheta}_{T}$ are defined as usual by the relations

$$
L\left(\hat{\vartheta}_{T}, X^{T}\right)=\sup _{\theta \in \Theta} L\left(\theta, X^{T}\right) \text { and } \tilde{\vartheta}_{T}=\frac{\int_{\alpha}^{\beta} \theta p(\theta) L\left(\theta, X^{T}\right) \mathrm{d} \theta}{\int_{\alpha}^{\beta} p(\theta) L\left(\theta, X^{T}\right) \mathrm{d} \theta} .
$$

To describe theirs properties we need the following notations. Let us introduce

- the random process

$$
Z_{0}(u)=\exp \left\{W(u)-\frac{|u|}{2}\right\}, \quad u \in \mathscr{R},
$$

where $W(\cdot)$ is two-sided Wiener process,

- two random variables $\hat{u}$ and $\tilde{u}$ defined by the relations

$$
Z_{0}(\hat{u})=\sup _{u \in \mathscr{R}} Z_{0}(u), \quad \tilde{u}=\frac{\int_{\mathscr{R}} u Z_{0}(u) \mathrm{d} u}{\int_{\mathscr{R}} Z_{0}(u) \mathrm{d} u}
$$

- the function

$$
\Gamma_{\vartheta}^{2}=\frac{\left(\rho_{2}-\rho_{1}\right)^{2} \vartheta^{2}}{G(\vartheta) \sigma^{2}} e^{-\frac{\rho_{1}^{2} \vartheta^{2}}{\sigma^{2}}} .
$$

The properties of estimators are given in the following proposition.

Proposition 1 Let the condition $\mathcal{A}$ be fulfilled, then the $M L E \hat{\vartheta}_{T}$ and the $B E \tilde{\vartheta}_{T}$ are uniformly on compacts $\mathbb{K} \subset \Theta$ consistent: for any $\nu>0$

$$
\sup _{\vartheta \in \mathbb{K}} \mathbf{P}_{\vartheta}\left\{\left|\hat{\vartheta}_{T}-\vartheta\right|>\nu\right\} \longrightarrow 0
$$

have two different limit distributions

$$
T\left(\hat{\vartheta}_{T}-\vartheta\right) \Longrightarrow \frac{\hat{u}}{\Gamma_{\vartheta}^{2}}, \quad T\left(\tilde{\vartheta}_{T}-\vartheta\right) \Longrightarrow \frac{\tilde{u}}{\Gamma_{\vartheta}^{2}},
$$


theirs moments converge: for any $p>0$

$$
\mathbf{E}_{\vartheta}\left|T\left(\hat{\vartheta}_{T}-\vartheta\right)\right|^{p} \longrightarrow \mathbf{E}\left|\frac{\hat{u}}{\Gamma_{\vartheta}^{2}}\right|^{p}, \quad \mathbf{E}_{\vartheta}\left|T\left(\tilde{\vartheta}_{T}-\vartheta\right)\right|^{p} \longrightarrow \mathbf{E}\left|\frac{\tilde{u}}{\Gamma_{\vartheta}^{2}}\right|^{p}
$$

For the proof see Section 6 .

Note that the same normalization and the same type limits (with different $\left.\Gamma_{\vartheta}\right)$ we have in the problem of delay $\vartheta$ estimation by the observations of the following Gaussian process

$$
\mathrm{d} X_{t}=-\rho X_{t-\vartheta} \mathrm{d} t+\sigma \mathrm{d} W_{t}, \quad 0 \leq t \leq T
$$

See details in [12] (or in [13], Section 3.3).

Remind that the Bayesian estimators are usually asymptotically efficient in singular parameter estimation problems [7]. The following lower bound is valid: for all estimators $\bar{\vartheta}_{T}$

$$
\varliminf_{\delta \rightarrow 0} \lim _{T \rightarrow \infty} \sup _{\left|\vartheta-\vartheta_{0}\right|<\delta} T^{2} \mathbf{E}_{\vartheta}\left(\bar{\vartheta}_{T}-\vartheta\right)^{2} \geq \frac{\mathbf{E} \tilde{u}^{2}}{\Gamma\left(\vartheta_{0}\right)^{4}}
$$

see [7], Section 1.9 (or [13], Proposition 2.24). We call an estimator $\vartheta_{T}^{*}$ asymptotically efficient if for all $\vartheta_{0} \in \Theta$ we have the equality

$$
\lim _{\delta \rightarrow 0} \lim _{T \rightarrow \infty} \sup _{\left|\vartheta-\vartheta_{0}\right|<\delta} T^{2} \mathbf{E}_{\vartheta}\left(\vartheta_{T}^{*}-\vartheta\right)^{2}=\frac{\mathbf{E} \tilde{u}^{2}}{\Gamma\left(\vartheta_{0}\right)^{4}}
$$

It can be verified that the convergence of the moments of bayesian estimators is uniform on the compacts in $\Theta$ and that the function $\gamma(\vartheta)$ is continuous. From these properties we obtain immediately the asymptotic efficiency of the bayesian estimators (in the sense of this lower bound).

The quantities $\mathbf{E} \hat{u}^{2}$ and $\mathbf{E} \tilde{u}^{2}$ were calculated by Terent'ev (1968) and Rubin and Song (1995) respectively

$$
\mathbf{E} \hat{u}^{2}=26>\mathbf{E} \tilde{u}^{2}=16 \zeta(3) \sim 19,3
$$

where $\zeta(\cdot)$ is Riemann zeta function. This relation shows the difference between the limit variances of the MLE and BE. 


\subsection{All parameters unknown}

It is possible to describe the properties of estimators in the case when all three parameters $\left(\rho_{1}, \rho_{2}, \vartheta\right)=\left(\vartheta_{1}, \vartheta_{2}, \vartheta_{3}\right)=\boldsymbol{\vartheta} \in \boldsymbol{\Theta}$ are unknown and we observe

$$
\mathrm{d} X_{t}=-\vartheta_{1} X_{t} \mathbb{I}_{\left\{X_{t}<\vartheta_{3}\right\}} \mathrm{d} t-\vartheta_{2} X_{t} \mathbb{I}_{\left\{X_{t} \geq \vartheta_{3}\right\}} \mathrm{d} t+\sigma \mathrm{d} W_{t}, \quad 0 \leq t \leq T .
$$

We have $\boldsymbol{\Theta}=\left(\alpha_{1}, \beta_{1}\right) \times\left(\alpha_{2}, \beta_{2}\right) \times\left(\alpha_{3}, \beta_{3}\right)$. Let us denote by $\xi$ the random variable with the density $f(\boldsymbol{\vartheta}, x)$.

Proposition 2 Suppose that $\beta_{1}<\alpha_{2}$ and $\alpha_{2}>0$, then the $M L E \hat{\boldsymbol{\vartheta}}_{T}, B E$ $\tilde{\boldsymbol{\vartheta}}_{T}$ are consistent, have the following limit distributions

$$
\begin{aligned}
\sqrt{T}\left(\hat{\vartheta}_{1, T}-\vartheta_{1}\right) & \Longrightarrow \zeta_{1} \sim \mathcal{N}\left(0, \frac{\sigma^{2}}{\mathbf{E}_{\vartheta} \xi^{2} \mathbb{I}_{\left\{\xi<\vartheta_{3}\right\}}}\right), \\
\sqrt{T}\left(\hat{\vartheta}_{2, T}-\vartheta_{2}\right) & \Longrightarrow \zeta_{2} \sim \mathcal{N}\left(0, \frac{\sigma^{2}}{\mathbf{E}_{\vartheta} \xi^{2} \mathbb{I}_{\left\{\xi \geq \vartheta_{3}\right\}}}\right), \\
T\left(\hat{\vartheta}_{3, T}-\vartheta_{3}\right) & \Longrightarrow \frac{\hat{u}}{\Gamma_{\vartheta}^{2}}, \quad T\left(\tilde{\vartheta}_{3, T}-\vartheta_{3}\right) \Longrightarrow \frac{\tilde{u}}{\Gamma_{\vartheta}^{2}}
\end{aligned}
$$

The $B E \tilde{\vartheta}_{1, T}, \tilde{\vartheta}_{2, T}$ have the same asymptotic properties as $\hat{\vartheta}_{1, T}, \hat{\vartheta}_{2, T}$, the random variables $\zeta_{1}$ and $\zeta_{2}$ are independent and are independent of $\hat{u}, \tilde{u}$.

The proof see in Section 6 .

The construction of the MLE can be slightly simplified by the following "separation".

The MLE of the first two components can be written as

$$
\hat{\vartheta}_{1, T}=-\frac{\int_{0}^{T} X_{t} \mathbb{1}_{\left\{X_{t}<\hat{\vartheta}_{3, T}\right\}} \mathrm{d} X_{t}}{\int_{0}^{T} X_{t}^{2} \mathbb{I}_{\left\{X_{t}<\hat{\vartheta}_{3, T}\right\}} \mathrm{d} t}, \quad \hat{\vartheta}_{2, T}=-\frac{\int_{0}^{T} X_{t} \mathbb{1}_{\left\{X_{t} \geq \hat{\vartheta}_{3, T}\right\}} \mathrm{d} X_{t}}{\int_{0}^{T} X_{t}^{2} \mathbb{I}_{\left\{X_{t}<\hat{\vartheta}_{3, T}\right\}} \mathrm{d} t}
$$

but to study these expressions can be quite difficult because the estimator $\hat{\vartheta}_{3, T}$ depends on the whole trajectory $X^{T}$ and therefore the random function $X_{t} \mathbb{1}_{\left\{X_{t}<\hat{\vartheta}_{3, T}\right\}}, \quad 0 \leq t \leq T$ depends of the "future". Hence the stochastic integral needs a special treatment. The problem can be simplified as follows. Let us estimate the parameter $\vartheta_{3}$ by the first $X^{\sqrt{T}}=\left\{X_{t}, 0 \leq t \leq \sqrt{T}\right\}$ observation and denote by $\vartheta_{3, \sqrt{T}}^{*}$ the corresponding consistent estimator. We suppose that there exists $b>0$ such that

$$
\mathbf{P}_{\vartheta}\left\{\left|\vartheta_{3, \sqrt{T}}^{*}-\vartheta_{3}\right|>T^{-b}\right\} \longrightarrow 0
$$


as $T \rightarrow \infty$. Then we define the estimators

$$
\hat{\vartheta}_{1, T}=-\frac{\int_{\sqrt{T}}^{T} X_{t} \mathbb{I}_{\left\{X_{t}<\vartheta_{3, \sqrt{T}}^{*}\right\}} \mathrm{d} X_{t}}{\int_{\sqrt{T}}^{T} X_{t}^{2} \mathbb{I}_{\left\{X_{t}<\vartheta_{3, \sqrt{T}}^{*}\right\}} \mathrm{d} t}, \quad \hat{\vartheta}_{2, T}=-\frac{\int_{\sqrt{T}}^{T} X_{t} \mathbb{I}_{\left\{X_{t} \geq \vartheta_{3, \sqrt{T}}^{*}\right\}} \mathrm{d} X_{t}}{\int_{\sqrt{T}}^{T} X_{t}^{2} \mathbb{I}_{\left\{X_{t} \geq \vartheta_{3, \sqrt{T}}^{*}\right\}} \mathrm{d} t} .
$$

Now the stochastic integrals are well defined and the consistency and asymptotic normality of these estimators follow from the usual limit theorems, i.e., we have

$$
\sqrt{T}\left(\hat{\vartheta}_{1, T}-\vartheta_{1}\right)=-\sigma \frac{\frac{1}{\sqrt{T}} \int_{\sqrt{T}}^{T} X_{t} \mathbb{I}_{\left\{X_{t}<\vartheta_{3, \sqrt{T}}^{*}\right\}} \mathrm{d} W_{t}}{\frac{1}{T} \int_{\sqrt{T}}^{T} X_{t}^{2} \mathbb{I}_{\left\{X_{t}<\vartheta_{3, \sqrt{T}}^{*}\right\}} \mathrm{d} t}
$$

with (law of large numbers)

$$
\frac{1}{T} \int_{\sqrt{T}}^{T} X_{t}^{2} \mathbb{I}_{\left\{X_{t}<\vartheta_{3, \sqrt{T}}^{*}\right\}} \mathrm{d} t \longrightarrow \mathbf{E}_{\theta} \xi^{2} \mathbb{I}_{\left\{\xi<\vartheta_{3}\right\}}
$$

and (central limit theorem)

$$
\frac{1}{\sqrt{T}} \int_{\sqrt{T}}^{T} X_{t} \mathbb{I}_{\left\{X_{t}<\vartheta_{3, \sqrt{T}}^{*}\right\}} \mathrm{d} W_{t} \Longrightarrow \zeta \sim \mathcal{N}\left(0, \mathbf{E}_{\theta} \xi^{2} \mathbb{I}_{\left\{\xi<\vartheta_{3}\right\}}\right) .
$$

hence

$$
\sqrt{T}\left(\hat{\vartheta}_{1, T}-\vartheta_{1}\right) \Longrightarrow \mathcal{N}\left(0, \frac{\sigma^{2}}{\mathbf{E}_{\theta} \xi^{2} \mathbb{I}_{\left\{\xi<\vartheta_{3}\right\}}}\right) .
$$

Note that the independence of the random variables $\zeta_{1}$ and $\zeta_{2}$ follows from the following property of stochastic integral

$$
\mathbf{E}_{\theta}\left(\int_{0}^{T} X_{t} \mathbb{I}_{\left\{X_{t}<\vartheta_{3}\right\}} \mathrm{d} W_{t} \int_{0}^{T} X_{t} \mathbb{I}_{\left\{X_{t} \geq \vartheta_{3}\right\}} \mathrm{d} W_{t}\right)=0 .
$$

The possibility to simplify the estimation of $\vartheta_{3}$ we discuss at the end of the next section.

\subsection{Misspecification}

Let us return to the initial problem of threshold estimation and suppose that the observed process is

$$
\mathrm{d} X_{t}=-\rho_{1} X_{t} \mathbb{I}_{\left\{X_{t}<\vartheta_{0}\right\}} \mathrm{d} t-\rho_{2} X_{t} \mathbb{I}_{\left\{X_{t} \geq \vartheta_{0}\right\}} \mathrm{d} t+h\left(X_{t}\right) \mathrm{d} t+\sigma \mathrm{d} W_{t},
$$

where $h(\cdot)$ is some unknown function (contamination) and $\vartheta_{0}$ is the true value. We assume that the statistician uses this model without $h(\cdot)$ (wrong 
model) and tries to estimate $\vartheta$, i.e., he (or she) supposes that the observed process is TOU (3) and construct, say, the MLE $\hat{\vartheta}_{T}$ as if $h(\cdot) \equiv 0$. Then he substitutes the observations (10) (of course, containing $h(\cdot)$. Such situation can be considered as typical for many applied problems, when there is a difference between the theoretical model and the real data. Remind that in regular case the MLE and BE are usually not consistent and converge to the value which minimizes the Kullback-Leibler distance (see [13], Section 2.6.1). The Kullback-Leibler distance in our problem is (suppose for instant that $\left.\vartheta_{0}<\vartheta\right)$

$$
\begin{aligned}
& D_{K-L}\left(\vartheta, \vartheta_{0}\right)=\mathbf{E}_{\vartheta_{0}}^{*} \ln \frac{\mathrm{d} \mathbf{P}_{\vartheta_{0}}^{*}}{\mathrm{~d} \mathbf{P}_{\vartheta}}\left(X^{T}\right) \\
& \quad=\frac{T}{2 \sigma^{2}} \mathbf{E}_{\vartheta_{0}}^{*}\left[\rho_{1} \xi\left[\mathbb{I}_{\{\xi<\vartheta\}}-\mathbb{I}_{\left\{\xi<\vartheta_{0}\right\}}\right]+\rho_{2} \xi\left[\mathbb{I}_{\{\xi \geq \vartheta\}}-\mathbb{1}_{\left\{\xi \geq \vartheta_{0}\right\}}\right]+h(\xi)\right]^{2} \\
& \quad=\frac{T}{2 \sigma^{2}} \mathbf{E}_{\vartheta_{0}}^{*}\left[\left(\rho_{1}-\rho_{2}\right) \xi \mathbb{I}_{\left\{\vartheta_{0}<\xi<\vartheta\right\}}+h(\xi)\right]^{2}
\end{aligned}
$$

where $\mathbf{E}_{\vartheta_{0}}^{*}$ denotes the expectation w.r.t. the measure $\mathbf{P}_{\vartheta_{0}}^{*}$ which corresponds to the process (10) (we denote its density as $f_{h}\left(\vartheta_{0}, x\right)$ ). It can be shown (see [13], Section 2.6.1) that

$$
\hat{\vartheta}_{T} \longrightarrow \vartheta_{*}=\arg \inf _{\vartheta \in \Theta} D_{K-L}\left(\vartheta, \vartheta_{0}\right) .
$$

We are interested by the following question: when $\vartheta_{*}=\vartheta_{0}$, i.e., when the MLE is nevertheless consistent? Surprisingly it is possible even for not too small functions $h(\cdot)$. Suppose, for simplicity, that $\vartheta \in \Theta=(\alpha, \beta), \alpha>0$.

Let us introduce the function

$$
K\left(\vartheta, \vartheta_{0}\right)= \begin{cases}\mathbf{E}_{\vartheta_{0}}^{*}\left[\left(\rho_{1}-\rho_{2}\right) \xi \mathbb{I}_{\left\{\vartheta_{0}<\xi<\vartheta\right\}}+h(\xi)\right]^{2}, & \text { if } \vartheta \geq \vartheta_{0} \\ \mathbf{E}_{\vartheta_{0}}^{*}\left[\left(\rho_{2}-\rho_{1}\right) \xi \mathbb{I}_{\left\{\vartheta<\xi<\vartheta_{0}\right\}}+h(\xi)\right]^{2}, & \text { if } \vartheta \leq \vartheta_{0}\end{cases}
$$

and suppose that $\rho_{2}>\rho_{1}$. Then for $\vartheta>\vartheta_{0}$ we have

$$
\begin{aligned}
K\left(\vartheta, \vartheta_{0}\right)= & {\left[\int_{-\infty}^{\vartheta_{0}}+\int_{\vartheta}^{\infty}\right] h(x)^{2} f_{h}\left(\vartheta_{0}, x\right) \mathrm{d} x } \\
& +\int_{\vartheta_{0}}^{\vartheta}\left[\left(\rho_{1}-\rho_{2}\right) x+h(x)\right]^{2} f_{h}\left(\vartheta_{0}, x\right) \mathrm{d} x,
\end{aligned}
$$

and

$$
\begin{aligned}
\frac{\partial K\left(\vartheta, \vartheta_{0}\right)}{\partial \vartheta} & =-h(\vartheta)^{2} f_{h}\left(\vartheta_{0}, \vartheta\right)+\left[\left(\rho_{1}-\rho_{2}\right) \vartheta+h(\vartheta)\right]^{2} f_{h}\left(\vartheta_{0}, \vartheta\right) \\
& =\left[\left(\rho_{1}-\rho_{2}\right)^{2} \vartheta^{2}+2\left(\rho_{1}-\rho_{2}\right) \vartheta h(\vartheta)\right] f_{h}\left(\vartheta_{0}, \vartheta\right)
\end{aligned}
$$


Therefore, if

$$
h(y)<\frac{y}{2}\left(\rho_{2}-\rho_{1}\right), \quad \text { for } \quad \alpha<y<\beta,
$$

then for $\vartheta>\vartheta_{0}$

$$
\frac{\partial K\left(\vartheta, \vartheta_{0}\right)}{\partial \vartheta}>0
$$

and similarly, if

$$
h(y)>-\frac{y}{2}\left(\rho_{2}-\rho_{1}\right), \quad \text { for } \quad \alpha<y<\beta,
$$

then for $\vartheta<\vartheta_{0}$

$$
\frac{\partial K\left(\vartheta, \vartheta_{0}\right)}{\partial \vartheta}<0
$$

We see that if the function $h(\cdot)$ satisfies the condition

$$
|h(y)|<\frac{y}{2}\left(\rho_{2}-\rho_{1}\right), \quad \alpha<y<\beta,
$$

then $\vartheta_{*}=\vartheta_{0}$ and the MLE $\hat{\vartheta}_{T}$ is consistent even for this "wrong model" (see [13], Section 3.4.5 for another example). Note, that there is no conditions on $h(y)$ for $y \notin[\alpha, \beta]$.

Let us return to the problem of the construction of the preliminary consistent estimator of the parameter $\vartheta_{3}$ by observations (6). Suppose that $\beta_{1}-\alpha_{1}<\alpha_{2}-\beta_{1}$ and $\beta_{2}-\alpha_{2}<\alpha_{2}-\beta_{1}$. Let us put

$$
\hat{\vartheta}_{1}=\frac{\alpha_{1}+\beta_{1}}{2}, \quad \hat{\vartheta}_{2}=\frac{\alpha_{2}+\beta_{2}}{2}
$$

and consider the problem of estimation $\vartheta_{3}$ by the "wrong model"

$$
\mathrm{d} X_{t}=-\hat{\vartheta}_{1} X_{t} \mathbb{1}_{\left\{X_{t}<\vartheta_{3}\right\}} \mathrm{d} t-\hat{\vartheta}_{2} X_{t} \mathbb{1}_{\left\{X_{t} \geq \vartheta_{3}\right\}} \mathrm{d} t+\sigma \mathrm{d} W_{t}, \quad 0 \leq t \leq \sqrt{T}
$$

with "known" $\hat{\vartheta}_{1}, \hat{\vartheta}_{2}$. This corresponds well to the model (10) with

$$
h(x)=\left(\hat{\vartheta}_{1}-\vartheta_{1}\right) x \mathbb{I}_{\left\{x<\vartheta_{3}\right\}}+\left(\hat{\vartheta}_{2}-\vartheta_{2}\right) x \mathbb{I}_{\left\{x \geq \vartheta_{3}\right\}} .
$$

We see that the condition (111) is fulfilled, hence the MLE $\hat{\vartheta}_{3, \sqrt{T}}$ is consistent and can be used in the construction of the estimators (8). Note that the estimator $\hat{\vartheta}_{3, \sqrt{T}}$ even has "singular" rate of convergence, but its limit distribution is different of that of the true MLE. 


\section{Other Threshold Models.}

Below we consider several other threshold type ergodic diffusion processes and discuss the properties of parameter estimators for these models.

\subsection{Simple Threshold model.}

Suppose that the observed process is

$$
\mathrm{d} X_{t}=\rho_{1} \mathbb{I}_{\left\{X_{t}<\vartheta\right\}} \mathrm{d} t-\rho_{2} \mathbb{I}_{\left\{X_{t} \geq \vartheta\right\}} \mathrm{d} t+\sigma \mathrm{d} W_{t}, \quad 0 \leq t \leq T,
$$

where $\rho_{i}>0$ and $\vartheta \in(\alpha, \beta)$. Then this process is ergodic with exponential type invariant density

$$
f(\vartheta, x)=\frac{1}{G(\vartheta)} \exp \left\{-\frac{2 \rho(x, \vartheta)|x-\vartheta|}{\sigma^{2}}\right\}
$$

where $\rho(x, \vartheta)=\rho_{1} \mathbb{I}_{\{x<\vartheta\}}+\rho_{2} \mathbb{I}_{\{x \geq \vartheta\}}$ and $G(\vartheta)$ is the normalizing constant.

The MLE $\hat{\vartheta}_{T}$ and BE $\tilde{\vartheta}_{T}$ have the same properties as in Theorem 1

$$
T\left(\hat{\vartheta}_{T}-\vartheta\right) \Longrightarrow \frac{\hat{u}}{\Gamma_{\vartheta}^{2}}, \quad T\left(\tilde{\vartheta}_{T}-\vartheta\right) \Longrightarrow \frac{\tilde{u}}{\Gamma_{\vartheta}^{2}}
$$

and the corresponding function

$$
\Gamma_{\vartheta}^{2}=\frac{2 \rho_{2} \rho_{1}\left(\rho_{2}+\rho_{1}\right)}{\sigma^{4}} .
$$

Note, that the normalized LR converges to the limit process as follows:

$$
Z_{T}(u)=\frac{L\left(\vartheta+\frac{u}{T}, X^{T}\right)}{L\left(\vartheta, X^{T}\right)} \Longrightarrow \exp \left\{\Gamma_{\vartheta} W(u)-\frac{|u|}{2} \Gamma_{\vartheta}^{2}\right\}
$$

The proof see in the Section 6.

\subsection{Simple Switching.}

Suppose that in the model (12) we have $\rho_{1}=\rho_{2}=\rho>0$. Then the observed process is

$$
\mathrm{d} X_{t}=-\rho \operatorname{sgn}\left(X_{t}-\vartheta\right) \mathrm{d} t+\sigma \mathrm{d} W_{t}, \quad 0 \leq t \leq T,
$$

where $\vartheta \in \Theta=(\alpha, \beta)$. This Simple Switching Process was studied in [13], Section 3.4.1. Remind that it has Laplace type invariant density

$$
f(\vartheta, x)=\frac{\rho}{\sigma^{2}} e^{-\frac{2 \rho}{\sigma^{2}}|x-\vartheta|} .
$$


The likelihood ratio formula has the representation

$$
L\left(\vartheta, X^{T}\right)=\exp \left\{-\frac{\rho}{\sigma^{2}} \int_{0}^{T} \operatorname{sgn}\left(X_{t}-\vartheta\right) \mathrm{d} X_{t}-\frac{\rho^{2} T}{2 \sigma^{2}}\right\} .
$$

Hence, the MLE $\hat{\vartheta}_{T}$ is defined by the equation

$$
\int_{0}^{T} \operatorname{sgn}\left(X_{t}-\hat{\vartheta}_{T}\right) \mathrm{d} X_{t}=\inf _{\vartheta \in(\alpha, \beta)} \int_{0}^{T} \operatorname{sgn}\left(X_{t}-\vartheta\right) \mathrm{d} X_{t} .
$$

Note that the last stochastic integral we find in Tanaka-Meyer representation of the local time of diffusion process (see [19])

$$
\Lambda_{T}(\vartheta)=\left|X_{T}-\vartheta\right|-\left|X_{0}-\vartheta\right|-\int_{0}^{T} \operatorname{sgn}\left(X_{t}-\vartheta\right) \mathrm{d} X_{t}
$$

and the maximum likelihood is in some sense asymptotically equivalent to the maximum local time estimator. Remind that $f_{T}^{\circ}(x)=\Lambda_{T}(x) / T \sigma^{2}$ is the consistent, asymptotically normal and asymptotically efficient (in nonparametric statement) estimator of the invariant density (see [13] for details), and we have obviously

$$
\sup _{\vartheta \in \Theta} f\left(\vartheta_{0}, \vartheta\right)=f\left(\vartheta_{0}, \vartheta_{0}\right)
$$

We have the same asymptotic properties of the MLE and BE as in the Theorem 1 .

The normalized LR

$$
Z_{T}(u)=\frac{L\left(\vartheta+\frac{u}{T}, X^{T}\right)}{L\left(\vartheta, X^{T}\right)} \Longrightarrow \exp \left\{\Gamma_{\vartheta} W(u)-\frac{|u|}{2} \Gamma_{\vartheta}^{2}\right\}, \quad \Gamma_{\vartheta}^{2}=\frac{4 \rho^{3}}{\sigma^{4}} .
$$

The proof can be found in [13], Section 3.4.

The observation window $(-\infty, \infty)$ can be essentially reduced. Let us put

$$
\vartheta_{\sqrt{T}}^{\star}=\frac{1}{\sqrt{T}} \int_{0}^{\sqrt{T}} X_{t} \mathrm{~d} t .
$$

Note that $\vartheta_{\sqrt{T}}^{\star}$ is an estimator of the method of moments $\left(\mathbf{E}_{\vartheta} \xi=\vartheta\right)$. It is consistent and asymptotically normal

$$
T^{1 / 4}\left(\vartheta_{\sqrt{T}}^{\star}-\theta\right) \Longrightarrow \mathcal{N}\left(0, d^{2}(\vartheta)\right)
$$


see [13], p. 270, where $d\left(\vartheta^{2}\right)$ is calculated. Introduce the window

$$
\mathbb{B}_{T}=\left[\vartheta_{\sqrt{T}}^{\star}-T^{-1 / 8}, \vartheta_{\sqrt{T}}^{\star}+T^{-1 / 8}\right] .
$$

The MLE and BE we define with the help of the $\operatorname{LR~} L\left(\vartheta, X_{\sqrt{T}}^{T}\right)$

$$
=\exp \left\{-\frac{\rho}{\sigma^{2}} \int_{\sqrt{T}}^{T} \operatorname{sgn}\left(X_{t}-\vartheta\right) \mathbb{I}_{\left\{X_{t} \in \mathbb{B}_{T}\right\}} \mathrm{d} X_{t}-\frac{\rho^{2}}{2 \sigma^{2}} \int_{\sqrt{T}}^{T} \mathbb{I}_{\left\{X_{t} \in \mathbb{B}_{T}\right\}} \mathrm{d} t\right\}
$$

Then these estimators have the same asymptotic properties as if the observation window is $\mathbb{B}_{T}=(-\infty, \infty)$.

This a bit surprising result is probably typical for singular estimation problems. The analyse of the proof of the properties of estimators (see [13], Section 3.4) shows that only the values of $X_{t}$ close to the true value $\vartheta_{0}$ have contribution to the limit likelihood ratio. Hence all other observations are irrelevant and can be deleted by introducing this window.

\subsection{Multy Threshold O-U Process.}

Suppose that the observed process is

$$
\mathrm{d} X_{t}=-\sum_{l=1}^{k+1} \rho_{l} X_{t} \mathbb{1}_{\left\{\vartheta_{l-1}<X_{t} \leq \vartheta_{l}\right\}} \mathrm{d} t+\sigma \mathrm{d} W_{t}, \quad 0 \leq t \leq T
$$

where $\rho_{1}>0, \rho_{k+1}>0, \rho_{l} \neq \rho_{m}>0, \vartheta_{0}=-\infty, \vartheta_{k+1}=\infty$ and $\boldsymbol{\vartheta}=$ $\left(\vartheta_{1}, \ldots, \vartheta_{k}\right) \in \boldsymbol{\Theta}=\Theta_{1} \times \ldots \times \Theta_{k}, \Theta_{l}=\left(\alpha_{l}, \beta_{l}\right), \beta_{l}<\alpha_{l+1}$. Then this process is ergodic and the normalized likelihood ratio $\left(\mathbf{u}=\left(u_{1}, \ldots, u_{k}\right)\right)$ has the following limit

$$
Z_{T}(\mathbf{u})=\frac{L\left(\boldsymbol{\vartheta}+\frac{\boldsymbol{u}}{T}, X^{T}\right)}{L\left(\boldsymbol{\vartheta}, X^{T}\right)} \Longrightarrow Z(\mathbf{u})=\prod_{l=1}^{k} \exp \left\{\Gamma_{l} W_{l}\left(u_{l}\right)-\frac{\left|u_{l}\right|}{2} \Gamma_{l}^{2}\right\}
$$

where $W_{l}(\cdot)$ are independent two-sided Wiener processes. The estimators $\hat{\boldsymbol{\vartheta}}_{T}=\left(\hat{\vartheta}_{1, T}, \ldots, \hat{\vartheta}_{k, T}\right)$ and $\tilde{\boldsymbol{\vartheta}}_{T}=\left(\tilde{\vartheta}_{1, T}, \ldots, \tilde{\vartheta}_{k, T}\right)$ are consistent, have asymptotically independent components,

$$
T\left(\hat{\vartheta}_{l, T}-\vartheta_{l}\right) \Longrightarrow \frac{\hat{u}_{l}}{\Gamma_{l}^{2}}, \quad T\left(\tilde{\vartheta}_{l, T}-\vartheta_{l}\right) \Longrightarrow \frac{\tilde{u}_{l}}{\Gamma_{l}^{2}},
$$

i.e.; $\left(\hat{u}_{l}, \tilde{u}_{l}\right)$ is independent on $\left(\hat{u}_{m}, \tilde{u}_{m}\right)$ if $l \neq m$ and the moments converge. The proof see in the section 6 . 


\section{General Threshold Model.}

Suppose that the observed diffusion process $X^{T}=\left\{X_{t}, 0 \leq t \leq T\right\}$ satisfies the equation

$$
\mathrm{d} X_{t}=\sum_{j=1}^{k+1} S_{j}\left(X_{t}\right) \mathbb{I}_{\left\{\vartheta_{j-1}<X_{t} \leq \vartheta_{j}\right\}} \mathrm{d} t+\sigma\left(X_{t}\right) \mathrm{d} W_{t}, \quad X_{0},
$$

where $\vartheta_{0}=-\infty, \vartheta_{j} \in \Theta_{j}=\left(\alpha_{j}, \beta_{j}\right), j=1, \ldots, k, \vartheta_{k+1}=\infty, \beta_{j}<\alpha_{j+1}$. The unknown parameter is $\boldsymbol{\vartheta}=\left(\vartheta_{1}, \ldots, \vartheta_{k}\right) \in \boldsymbol{\Theta}=\Theta_{1} \times \ldots \times \Theta_{k}$. Our goal is to estimate $\boldsymbol{\vartheta}$ and to describe the asymptotic properties of estimators as $T \rightarrow \infty$. As before, we are interested by the estimators obtained by the Maximum likelihood and Bayesian methods.

This model can be called "Nonlinear Threshold Diffusion Process". Of course, all considered above models are nonlinear due to the indicator functions. Here we use the term "nonlinear" because the linear function $\rho x$ in the trend coefficient $-\rho x \mathbb{1}_{\{\cdot\}}$ is replaced by more general function $S(x)$.

$\mathcal{E S}$. The functions $S_{j}(\cdot)$ are locally bounded, the function $\sigma(\cdot)^{2}$ is continuous and positive and for some $A>0$ the condition

$$
x S_{1}(x) \mathbb{I}_{\left\{x<\alpha_{1}\right\}}+x S_{k+1}(x) \mathbb{I}_{\left\{x \geq \beta_{k}\right\}}+\sigma(x)^{2} \leq A\left(1+x^{2}\right)
$$

holds

This condition provides the existence of unique weak solution (see [4]).

We suppose that all measures $\left\{\mathbf{P}_{\boldsymbol{\vartheta}}^{(T)}, \boldsymbol{\vartheta} \in \boldsymbol{\Theta}\right\}$ induced by this process in the space $(\mathcal{C}(0, T), \mathcal{B}(0, T))$ are equivalent to the measure $\mathbf{P}^{(T)}$, which corresponds to the process

$$
\mathrm{d} X_{t}=\sigma\left(X_{t}\right) \mathrm{d} W_{t}, \quad X_{0}, \quad 0 \leq t \leq T
$$

(see [16]). The likelihood ratio

$$
L\left(\boldsymbol{\vartheta}, X^{T}\right)=\frac{\mathrm{d} \mathbf{P}_{\boldsymbol{\vartheta}}^{(T)}}{\mathrm{d} \mathbf{P}^{(T)}}\left(X^{T}\right), \quad \boldsymbol{\vartheta} \in \boldsymbol{\Theta},
$$

in this problem is the random function

$$
\begin{aligned}
& \ln L\left(\boldsymbol{\vartheta}, X^{T}\right)=\sum_{j=1}^{k+1} \int_{0}^{T} \frac{S_{j}\left(X_{t}\right)}{\sigma\left(X_{t}\right)^{2}} \mathbb{I}_{\left\{\vartheta_{j-1}<X_{t} \leq \vartheta_{j}\right\}} \mathrm{d} X_{t} \\
& \quad-\sum_{j=1}^{k+1} \int_{0}^{T} \frac{S_{j}\left(X_{t}\right)^{2}}{2 \sigma\left(X_{t}\right)^{2}} \mathbb{I}_{\left\{\vartheta_{j-1}<X_{t} \leq \vartheta_{j}\right\}} \mathrm{d} t .
\end{aligned}
$$


The MLE $\widehat{\boldsymbol{\vartheta}}_{T}$ is defined by the same equation

$$
L\left(\widehat{\boldsymbol{\vartheta}}_{T}, X^{T}\right)=\sup _{\boldsymbol{\theta} \in \boldsymbol{\Theta}} L\left(\boldsymbol{\theta}, X^{T}\right),
$$

where the function $L\left(\boldsymbol{\vartheta}, X^{T}\right)$ is not differentiable with respect to $\boldsymbol{\vartheta}$.

Note that

$$
\mathbb{I}_{\left\{\vartheta_{j-1}<x \leq \vartheta_{j}\right\}}=\mathbb{1}_{\left\{x \leq \vartheta_{j}\right\}}-\mathbb{I}_{\left\{x \leq \vartheta_{j-1}\right\}}
$$

Hence

$$
\begin{aligned}
\sum_{j=1}^{k+1} S_{j}(x) \mathbb{1}_{\left\{\vartheta_{j-1}<x \leq \vartheta_{j}\right\}} & =\sum_{j=1}^{k+1} S_{j}(x) \mathbb{1}_{\left\{x \leq \vartheta_{j}\right\}}-\sum_{j=1}^{k+1} S_{j}(x) \mathbb{1}_{\left\{x \leq \vartheta_{j-1}\right\}} \\
& =S_{k+1}(x)+\sum_{j=1}^{k}\left[S_{j}(x)-S_{j+1}(x)\right] \mathbb{1}_{\left\{x \leq \vartheta_{j}\right\}}
\end{aligned}
$$

and we can write the likelihood ratio as product of $k+1$ "likelihood ratios"

$$
\hat{L}\left(\boldsymbol{\vartheta}, X^{T}\right)=\frac{\mathrm{d} \mathbf{P}_{\vartheta}^{(T)}}{\mathrm{d} \mathbf{P}_{0}^{(T)}}\left(X^{T}\right)=L_{k+1}\left(X^{T}\right) \prod_{j=1}^{k} L_{j}\left(\vartheta_{j}, X^{T}\right)
$$

where

$$
\ln L_{k+1}\left(X^{T}\right)=\int_{0}^{T} \frac{S_{k+1}\left(X_{t}\right)}{\sigma\left(X_{t}\right)^{2}} \mathrm{~d} X_{t}-\int_{0}^{T} \frac{S_{k+1}\left(X_{t}\right)^{2}}{2 \sigma\left(X_{t}\right)^{2}} \mathrm{~d} t
$$

and

$$
\begin{aligned}
\ln L_{j}\left(\vartheta_{j}, X^{T}\right)=\int_{0}^{T} \frac{S_{j}\left(X_{t}\right)-S_{j+1}\left(X_{t}\right)}{\sigma\left(X_{t}\right)} \mathbb{I}_{\left\{X_{t} \leq \vartheta_{j}\right\}} \mathrm{d} X_{t} \\
\\
\quad-\int_{0}^{T} \frac{\left[S_{j}\left(X_{t}\right)^{2}-S_{j+1}\left(X_{t}\right)^{2}\right]}{2 \sigma\left(X_{t}\right)^{2}} \mathbb{I}_{\left\{X_{t} \leq \vartheta_{j}\right\}} \mathrm{d} t .
\end{aligned}
$$

This allows us to reduce the calculation of the MLE $\widehat{\boldsymbol{\vartheta}}_{T}$ of multidimensional parameter $\boldsymbol{\vartheta}$ to $k$ one-dimensional problems :

$$
\hat{\vartheta}_{j, T}=\operatorname{argmax}_{\vartheta_{j} \in \Theta_{j}} L_{j}\left(\vartheta_{j}, X^{T}\right), \quad j=1, \ldots, k,
$$

and to put $\widehat{\boldsymbol{\vartheta}}_{T}=\left(\hat{\vartheta}_{1, T}, \ldots, \hat{\vartheta}_{k, T}\right)$.

To introduce the Bayesian estimator $\tilde{\boldsymbol{\vartheta}}_{T}$ we suppose that $\boldsymbol{\vartheta}$ is a random vector with a known continuous positive density a priori $p(\boldsymbol{\theta}), \boldsymbol{\theta} \in \boldsymbol{\Theta}$ and the 
loss function $\ell(\boldsymbol{u}), \boldsymbol{u} \in \mathscr{R}^{k}$ is strictly convex. The estimator $\widetilde{\boldsymbol{\vartheta}}_{T}$ is defined as solution of the following equation

$$
\int_{\boldsymbol{\Theta}} \mathbf{E}_{\boldsymbol{\theta}} \ell\left(\widetilde{\boldsymbol{\vartheta}}_{T}-\boldsymbol{\theta}\right) p(\boldsymbol{\theta}) \mathrm{d} \boldsymbol{\theta}=\inf _{\boldsymbol{\vartheta} \in \boldsymbol{\Theta}} \int_{\boldsymbol{\Theta}} \mathbf{E}_{\boldsymbol{\theta}} \ell(\boldsymbol{\vartheta}-\boldsymbol{\theta}) p(\boldsymbol{\theta}) \mathrm{d} \boldsymbol{\theta} .
$$

Remind that in the case $\ell(\boldsymbol{u})=|\boldsymbol{u}|^{2}$ this estimator is

$$
\widetilde{\boldsymbol{\vartheta}}_{T}=\frac{\int_{\boldsymbol{\Theta}} \boldsymbol{\theta} L\left(\boldsymbol{\theta}, X^{T}\right) p(\boldsymbol{\theta}) \mathrm{d} \boldsymbol{\theta}}{\int_{\Theta} L\left(\boldsymbol{\theta}, X^{T}\right) p(\boldsymbol{\theta}) \mathrm{d} \boldsymbol{\theta}} .
$$

In this case we can simplify the calculation of the estimator too. Suppose that the density $p(\boldsymbol{\theta})=p_{1}\left(\theta_{1}\right) \cdots p_{k}\left(\theta_{k}\right)$ (the components of $\boldsymbol{\vartheta}$ are independent random variables). Then, using (17), we can write

$$
\tilde{\vartheta}_{j, T}=\frac{\int_{\Theta_{j}} \theta_{j} L_{j}\left(\theta_{j}, X^{T}\right) p_{j}\left(\theta_{j}\right) \mathrm{d} \theta_{j}}{\int_{\Theta_{j}} L_{j}\left(\theta_{j}, X^{T}\right) p_{j}\left(\theta_{j}\right) \mathrm{d} \theta_{j}}, \quad j=1, \ldots, k,
$$

and then to put $\widetilde{\boldsymbol{\vartheta}}_{T}=\left(\tilde{\vartheta}_{1, T}, \ldots, \tilde{\vartheta}_{k, T}\right)$.

The asymptotic behavior of the diffusion process is defined by the following condition.

$\mathcal{A}$. The functions $S_{1}(x), S_{k+1}(x)$ and $\sigma(x)$ satisfy the conditions

$$
|\sigma(x)|^{-1} \leq B\left(1+|x|^{m}\right)
$$

with some $B>0$ and $m>0$ and

$$
\varliminf_{x \rightarrow-\infty} \frac{S_{1}(x)}{\sigma(x)^{2}}>0, \quad \varlimsup_{x \rightarrow \infty} \frac{S_{k+1}(x)}{\sigma(x)^{2}}<0 .
$$

By this condition the process $\left(X_{t}\right)_{t \geq 0}$ has ergodic properties. Let us denote by $f(\boldsymbol{\vartheta}, x)$ the density of its invariant law and by $\xi$ the random variable with such density function. Note that by this condition $\xi$ has all polynomial moments [13].

The identifiability condition in this statistical problem is the following one

$$
\inf _{y \in\left(\alpha_{j}, \beta_{j}\right)}\left|S_{j}(y)-S_{j+1}(y)\right|>0, \quad j=1, \ldots, k .
$$

Let us introduce $\hat{\boldsymbol{u}}_{\vartheta}=\left(\hat{u}_{1, \vartheta}, \ldots, \hat{u}_{k, \vartheta}\right)$, where

$$
\hat{u}_{j, \boldsymbol{\vartheta}}=\frac{\hat{u}_{j}}{\gamma_{j}(\boldsymbol{\vartheta})}, \quad \gamma_{j}(\boldsymbol{\vartheta})^{2}=\frac{\left(S_{j+1}\left(\vartheta_{j}\right)-S_{j}\left(\vartheta_{j}\right)\right)^{2}}{\sigma\left(\vartheta_{j}\right)^{2}} f\left(\boldsymbol{\vartheta}, \vartheta_{j}\right)
$$


and $\hat{u}_{1}, \ldots, \hat{u}_{k}$ are independent random variables defined by the equalities

$$
\hat{u}_{j}=\operatorname{argsup}_{u \in \mathscr{R}}\left[W_{j}(u)-\frac{1}{2}|u|\right] .
$$

Here $W_{j}(\cdot), j=1, \ldots, k$ are independent two-sided Wiener processes.

Let us define the random vector $\tilde{\boldsymbol{u}}_{\vartheta}$ as solution of the following equation

$$
\int_{\mathscr{R}^{k}} \ell\left(\tilde{\boldsymbol{u}}_{\vartheta}-\boldsymbol{u}\right) Z(\boldsymbol{u}) \mathrm{d} \boldsymbol{u}=\inf _{\boldsymbol{v} \in \mathscr{R}^{k}} \int_{\mathscr{R}^{k}} \ell(\boldsymbol{v}-\boldsymbol{u}) Z(\boldsymbol{u}) \mathrm{d} \boldsymbol{u},
$$

where

$$
Z(\boldsymbol{u})=\exp \left\{\sum_{j=1}^{k}\left[\gamma_{j}(\boldsymbol{\vartheta}) W_{j}\left(u_{j}\right)-\frac{\left|u_{j}\right|}{2} \gamma_{j}(\boldsymbol{\vartheta})^{2}\right]\right\}
$$

Theorem 1 Suppose that these conditions $\mathcal{E S}, \mathcal{A}$ and (18) are fulfilled, then the MLE $\widehat{\boldsymbol{\vartheta}}_{T}$ and bayessian estimator $\widetilde{\boldsymbol{\vartheta}}_{T}$ are consistent, have the following limit distributions:

$$
T\left(\widehat{\boldsymbol{\vartheta}}_{T}-\boldsymbol{\vartheta}\right) \Longrightarrow \hat{\boldsymbol{u}}_{\boldsymbol{\vartheta}}, \quad T\left(\widetilde{\boldsymbol{\vartheta}}_{T}-\boldsymbol{\vartheta}\right) \Longrightarrow \tilde{\boldsymbol{u}}_{\boldsymbol{\vartheta}}
$$

and the moments converge : for any $p>0$

$$
\lim _{T \rightarrow \infty} T^{p} \mathbf{E}_{\vartheta}\left|\widehat{\boldsymbol{\vartheta}}_{T}-\vartheta\right|^{p}=\mathbf{E}\left|\hat{\boldsymbol{u}}_{\vartheta}\right|^{p}, \quad \lim _{T \rightarrow \infty} T^{p} \mathbf{E}_{\vartheta}\left|\tilde{\vartheta}_{T}-\vartheta\right|^{p}=\mathbf{E}\left|\tilde{\boldsymbol{u}}_{\vartheta}\right|^{p}
$$

The proof is given in the section 6 .

\section{Proofs}

First note that the parameter estimation problems for the models of the observations (3), (12) and (13) are particular cases of the threshold estimation problem for stochastic process (15). Therefore, it is sufficient to prove the Theorem 1 .

The proof of this theorem is based on the two remarkable theorems by Ibragimov and Khasminskii ([7], Theorems 1.10.1 and 1.10.2) and some results obtained before in [13]. Let us remind the main steps of this approach. Introduce the random function (normalized likelihood ratio)

$$
Z_{T}(\boldsymbol{u})=\frac{L\left(\boldsymbol{\vartheta}+\frac{\boldsymbol{u}}{T}, X^{T}\right)}{L\left(\boldsymbol{\vartheta}, X^{T}\right)}, \quad \boldsymbol{u} \in \boldsymbol{U}_{T}=U_{1, T} \times \ldots U_{k, T},
$$


where $U_{j, T}=\left(T\left(\alpha_{j}-\vartheta_{j}\right), T\left(\beta_{j}-\vartheta_{j}\right)\right)$. The properties of estimators follow, roughly speaking, from the weak convergence of this function to the limit random field (19):

$$
Z_{T}(\boldsymbol{u})=\frac{L\left(\boldsymbol{\vartheta}+\frac{\boldsymbol{u}}{T}, X^{T}\right)}{L\left(\boldsymbol{\vartheta}, X^{T}\right)} \Longrightarrow Z(\boldsymbol{u})
$$

Suppose that we have already this convergence and (for simplicity) assume that $k=1$. Then for the MLE we have ( $\vartheta$ is the true value) :

$$
\begin{aligned}
\mathbf{P}_{\vartheta} & \left\{T\left(\hat{\vartheta}_{T}-\vartheta\right)<x\right\}= \\
& =\mathbf{P}\left\{\sup _{T(\theta-\vartheta)<x} L\left(\theta, X^{T}\right)>\sup _{T(\theta-\vartheta) \geq x} L\left(\theta, X^{T}\right)\right\} \\
& =\mathbf{P}\left\{\sup _{T(\theta-\vartheta)<x} \frac{L\left(\vartheta, X^{T}\right)}{L\left(\vartheta, X^{T}\right)}>\sup _{T(\theta-\vartheta) \geq x} \frac{L\left(\vartheta, X^{T}\right)}{L\left(\vartheta_{0}, X^{T}\right)}\right\} \\
& =\mathbf{P}\left\{\sup _{u<x} Z_{T}(u)>\sup _{u \geq x} Z_{T}(u)\right\} \\
& =\mathbf{P}\left(\frac{\hat{u}}{\gamma(\vartheta)^{2}}<x\right), \quad \text { i.e. } T\left(\sup _{u<x} Z(u)>\sup _{u \geq x} Z(u)\right\}
\end{aligned}
$$

where we put $\theta=\vartheta+T^{-1} u$.

To describe the behavior of the BE we take we for simplicity the square loss function and use the same change of variables $\theta=\vartheta+u / T \equiv \theta_{u}$ and ,

$$
\begin{aligned}
& \tilde{\vartheta}_{T}=\frac{\int_{\alpha}^{\beta} \theta p(\theta) L\left(\theta, X^{T}\right) \mathrm{d} \theta}{\int_{\alpha}^{\beta} p(\theta) L\left(\theta, X^{T}\right) \mathrm{d} \theta}=\vartheta+\frac{1}{T} \frac{\int_{U_{T}} u p\left(\theta_{u}\right) L\left(\theta_{u}, X^{T}\right) \mathrm{d} u}{\int_{U_{T}} p\left(\theta_{u}\right) L\left(\theta_{u}, X^{T}\right) \mathrm{d} u} \\
& =\vartheta+\frac{1}{T} \frac{\int_{U_{T}} u p\left(\theta_{u}\right) \frac{L\left(\theta_{u}, X^{T}\right)}{L\left(\vartheta, X^{T}\right)} \mathrm{d} u}{\int_{U_{T}} p\left(\theta_{u}\right) \frac{L\left(\theta_{u}, X^{T}\right)}{L\left(\vartheta, X^{T}\right)} \mathrm{d} u}=\vartheta+\frac{1}{T} \frac{\int_{U_{T}} u p\left(\theta_{u}\right) Z_{T}(u) \mathrm{d} u}{\int_{U_{T}} p\left(\theta_{u}\right) Z_{T}(u) \mathrm{d} u}
\end{aligned}
$$

Then, using the convergence $p\left(\theta_{u}\right) \rightarrow p(\vartheta)$, we can write

$$
\begin{aligned}
\mathbf{P}_{\vartheta}\left\{T\left(\tilde{\vartheta}_{T}-\vartheta\right)\right. & <x\}=\mathbf{P}\left\{\frac{\int_{U_{T}} u p\left(\theta_{u}\right) Z_{T}(u) \mathrm{d} u}{\int_{U_{T}} p\left(\theta_{u}\right) Z_{T}(u) \mathrm{d} u}<x\right\} \\
\longrightarrow \mathbf{P} & \left\{\frac{\int_{R} u Z(u) \mathrm{d} u}{\int_{R} Z(u) \mathrm{d} u}<x\right\}=\mathbf{P}\left(\frac{\tilde{u}}{\gamma(\vartheta)^{2}}<x\right) .
\end{aligned}
$$


The random variables $\hat{u}$ and $\tilde{u}$ are defined in (5).

We see that to prove the theorem we need to prove the convergences (20), (21). These convergences together with the estimates on the large deviations of estimators will provide the convergence of moments. The corresponding sufficient conditions are given in the mentioned above theorems by Ibragimov and Khasminskii. Let us introduce the conditions

A. The finite dimensional distributions of the random function $Z_{T}(\cdot)$ converge to the finite dimensional distributions of the function $Z(\cdot)$.

B. There exist constants $B>0, m>0, b>0$ and $d$ such that for any $R>0$ and $|\boldsymbol{u}| \leq R,|\boldsymbol{v}| \leq R$

$$
\mathbf{E}_{\boldsymbol{\vartheta}}\left|Z_{T}^{\frac{1}{2 m}}(\boldsymbol{u})-Z_{T}^{\frac{1}{2 m}}(\boldsymbol{v})\right|^{2 m} \leq B\left(1+R^{b}\right)|\boldsymbol{u}-\boldsymbol{v}|^{d} .
$$

C. For any $N>0$, there exists constant $C_{N}>0$, such that

$$
\mathbf{E}_{\vartheta} Z_{T}^{\frac{1}{2}}(\boldsymbol{u}) \leq \frac{C_{N}}{|\boldsymbol{u}|^{N}}
$$

These conditions are the version of the conditions of Theorems 1.10.1 (with $d>k$ ) and 1.10.2 [7, which we will verify in this work.

We start with the condition A. Let us consider the case when all $u_{j}>0$ and denote $h_{j}(x)=S_{j}(x) / \sigma(x)$. Note that

$$
\begin{aligned}
& \mathbb{I}_{\left\{\vartheta_{j-1}+\frac{\left.u_{j-1}<X_{t} \leq \vartheta_{j}+\frac{u_{j}}{T}\right\}}{T}-\mathbb{I}_{\left\{\vartheta_{j-1}<X_{t} \leq \vartheta_{j}\right\}}\right.} \\
& \quad=\mathbb{I}_{\left\{\vartheta_{j}<X_{t} \leq \vartheta_{j}+\frac{u_{j}}{T}\right\}}-\mathbb{I}_{\left\{\vartheta_{j-1}<X_{t} \leq \vartheta_{j-1}+\frac{u_{j-1}}{T}\right\}}=\mathbb{I}_{\left\{\mathbb{B}_{j}\right\}}-\mathbb{I}_{\left\{\mathbb{B}_{j-1}\right\}}
\end{aligned}
$$

in obvious notation.

Then the likelihood ratio $Z_{T}(\boldsymbol{u})$ can be written as follows

$$
\begin{aligned}
\ln Z_{T}(\boldsymbol{u})= & \sum_{j=1}^{k+1} \int_{0}^{T} h_{j}\left(X_{t}\right)\left[\mathbb{I}_{\left\{\mathbb{B}_{j}\right\}}-\mathbb{I}_{\left\{\mathbb{B}_{j-1}\right\}}\right] \mathrm{d} W_{t} \\
& -\frac{1}{2} \sum_{j=1}^{k} \int_{0}^{T}\left[h_{j}\left(X_{t}\right)-h_{j+1}\left(X_{t}\right)\right]^{2} \mathbb{I}_{\left\{\mathbb{B}_{j}\right\}} \mathrm{d} t .
\end{aligned}
$$

Using the local time estimator $f_{T}^{\circ}(x)$ of the invariant density $f(\boldsymbol{\vartheta}, x)$ we 
write

$$
\begin{aligned}
\int_{0}^{T} & {\left[h_{j}\left(X_{t}\right)-h_{j+1}\left(X_{t}\right)\right]^{2} \mathbb{I}_{\left\{\mathbb{B}_{j}\right\}} \mathrm{d} t } \\
= & T \int_{-\infty}^{\infty}\left[h_{j}(x)-h_{j+1}(x)\right]^{2} \mathbb{I}_{\left\{\vartheta_{j}<x \leq \vartheta_{j}+\frac{u_{j}}{T}\right\}} f_{T}^{\circ}(x) \mathrm{d} x \\
= & T \int_{\vartheta_{j}}^{\vartheta_{j}+\frac{u_{j}}{T}}\left[h_{j}(x)-h_{j+1}(x)\right]^{2} f_{T}^{\circ}(x) \mathrm{d} x \\
= & T \int_{\vartheta_{j}}^{\vartheta_{j}+\frac{u_{j}}{T}}\left[h_{j}(x)-h_{j+1}(x)\right]^{2} f(\boldsymbol{\vartheta}, x) \mathrm{d} x \\
& +T \int_{\vartheta_{j}}^{\vartheta_{j}+\frac{u_{j}}{T}}\left[h_{j}(x)-h_{j+1}(x)\right]^{2}\left[f_{T}^{\circ}(x)-f(\boldsymbol{\vartheta}, x)\right] \mathrm{d} x .
\end{aligned}
$$

For the random function $\eta_{T}(x)=T\left(f_{T}^{\circ}(x)-f(\boldsymbol{\vartheta}, x)\right)$ we have the estimate: for any $p>0$ there exist constants $C_{*}>0$ and $c_{*}>0$ such that

$$
\mathbf{E}_{\vartheta}\left|\eta_{T}(x)\right|^{p} \leq C_{*} e^{-c_{*}|x|}
$$

see Proposition 1.11 in [13]. This estimate allows us to prove that the last integral tends to zero as $T \rightarrow \infty$. We have as well

$$
\begin{aligned}
T \int_{\vartheta_{j}}^{\vartheta_{j}+\frac{u_{j}}{T}} & {\left[h_{j}(x)-h_{j+1}(x)\right]^{2} f(\boldsymbol{\vartheta}, x) \mathrm{d} x } \\
& \longrightarrow u_{j}\left[h_{j}\left(\vartheta_{j}\right)-h_{j+1}\left(\vartheta_{j}\right)\right]^{2} f\left(\boldsymbol{\vartheta}, \vartheta_{j}\right)=u_{j} \gamma_{j}(\boldsymbol{\vartheta})^{2} .
\end{aligned}
$$

Therefore,

$$
\sum_{j=1}^{k} \int_{0}^{T}\left[h_{j}\left(X_{t}\right)-h_{j+1}\left(X_{t}\right)\right]^{2} \mathbb{I}_{\left\{\mathbb{B}_{j}\right\}} \mathrm{d} t \longrightarrow \sum_{j=1}^{k} u_{j} \gamma_{j}(\boldsymbol{\vartheta})^{2} .
$$

This convergence by the central limit theorem for stochastic integrals yields the asymptotic normality of the vector $\boldsymbol{\xi}_{T}=\left(\xi_{1, T}, \ldots, \xi_{k, T}\right)$

$$
\xi_{j, T}=\int_{0}^{T}\left[h_{j}\left(X_{t}\right)-h_{j+1}\left(X_{t}\right)\right] \mathbb{I}_{\left\{\mathbb{B}_{j}\right\}} \mathrm{d} W_{t} \Longrightarrow \mathcal{N}\left(0, u_{j} \gamma_{j}(\boldsymbol{\vartheta})^{2}\right)
$$

with asymptotically independent components, because

$$
\mathbf{E}_{\vartheta} \xi_{j, T} \xi_{l, T}=0, \quad l \neq j .
$$


Moreover, if we put $\xi_{j, T}=\xi_{j, T}\left(u_{j}\right)$ and consider the vector $\boldsymbol{\xi}_{j, T}=\left(\xi_{j, T}\left(u_{j, 1}\right)\right.$, $\left.\ldots, \xi_{j, T}\left(u_{j, n}\right)\right)$, where $u_{j, 1}, \ldots, u_{j, n}$ is some collection of values from $U_{j, T}$, then

$$
\begin{aligned}
\mathbf{E}_{\vartheta} \xi_{j, T}\left(u_{j, r}\right) \xi_{j, T}\left(u_{j, q}\right)=T & \int_{\vartheta_{j}}^{\vartheta_{j}+\frac{u_{j, r} \wedge u_{j, q}}{T}}\left[h_{j}(x)-h_{j+1}(x)\right]^{2} f(\boldsymbol{\vartheta}, x) \mathrm{d} x \\
& \longrightarrow\left[u_{j, r} \wedge u_{j, q}\right] \gamma_{j}(\boldsymbol{\vartheta})^{2} .
\end{aligned}
$$

Using this equality and preceding limits we can show the convergence

$$
\left(\xi_{j, T}\left(u_{j, 1}\right), \ldots, \xi_{j, T}\left(u_{j, n}\right)\right) \Longrightarrow \gamma_{j}(\boldsymbol{\vartheta})\left(W_{j}\left(u_{j, 1}\right), \ldots, W_{j}\left(u_{j, 1}\right)\right) .
$$

Therefore the condition $\mathbf{A}$ is fulfilled.

To verify $\mathbf{B}$ we do it twice. The first time we check this condition with $m=1$, which is sufficient for Bayes estimators (multidimensional case) and then (for MLE) we verify it for the partial likelihoods $Z_{j, T}(u)$. Following [13], Lemma 3.28 we write (we suppose that $v_{j}<u_{j}$ )

$$
\begin{aligned}
& \mathbf{E}_{\vartheta}\left|Z_{T}^{1 / 2}(\boldsymbol{u})-Z_{T}^{1 / 2}(\boldsymbol{v})\right|^{2} \leq \frac{1}{4} \sum_{j=1}^{k} \mathbf{E}_{*} \int_{0}^{T}\left[h_{j}\left(X_{t}\right)-h_{j+1}\left(X_{t}\right)\right]^{2} \mathbb{I}_{\left\{\tilde{\mathbb{B}}_{j}\right\}} \mathrm{d} t \\
& =\frac{1}{4} \sum_{j=1}^{k} T \int_{-\infty}^{\infty}\left[h_{j}(x)-h_{j+1}(x)\right]^{2} \mathbb{I}_{\left\{\vartheta_{j}+\frac{v_{j}}{T}<x \leq \vartheta_{j}+\frac{u_{j}}{T}\right\}} f_{*}(x) \mathrm{d} x \\
& =\frac{1}{4} \sum_{j=1}^{k} T \int_{\vartheta_{j}+\frac{v j}{T}}^{\vartheta_{j}+\frac{u_{j}}{T}}\left[h_{j}(x)-h_{j+1}(x)\right]^{2} f_{*}(x) \mathrm{d} x \\
& \quad \leq C \sum_{j=1}^{k}\left|u_{j}-v_{j}\right| \leq C\|\boldsymbol{u}-\boldsymbol{v}\| .
\end{aligned}
$$

Here $\mathbf{E}_{*}$ and $f_{*}(\cdot)$ are expectation and invariant density which correspond to the stochastic differential equation

$$
\begin{gathered}
\mathrm{d} X_{t}=\sum_{j=1}^{k+1} S_{j}\left(X_{t}\right)\left[\mathbb{I}_{\left\{\vartheta_{j-1}+\frac{u_{j-1}}{T}<X_{t} \leq \vartheta_{j}+\frac{u_{j}}{T}\right\}}+\mathbb{I}_{\left\{\vartheta_{j-1}+\frac{v_{j-1}}{T}<X_{t} \leq \vartheta_{j}+\frac{v_{j}}{T}\right\}}\right] \mathrm{d} t \\
+\sigma\left(X_{t}\right) \mathrm{d} W_{t}, \quad X_{0}, \quad 0 \leq t \leq T
\end{gathered}
$$

(see details in [13], p. 379). The notation $\tilde{\mathbb{B}}_{j}$ is clear from the second line of (25).

The condition $\mathbf{B}$ in the case of the study the MLE we check for the components $Z_{j, T}\left(u_{j}\right), u_{j} \in \mathbf{U}_{j, T}$ separately as follows. Let us introduce the 
stochastic process

$$
V_{j, t}=\left(\frac{Z_{j, t}\left(u_{j}\right)}{Z_{j, t}\left(v_{j}\right)}\right)^{1 / 16}, \quad V_{j, 0}=1, \quad 0 \leq t \leq T
$$

and denote

$$
g_{j}(x)=\frac{S_{j}(x)-S_{j+1}(x)}{\sigma(x)}
$$

Then the process

$$
\begin{aligned}
V_{j, t}=\exp \left\{\frac{1}{16} \int_{0}^{t} \frac{S_{j}\left(X_{s}\right)-S_{j+1}\left(X_{s}\right)}{\sigma\left(X_{s}\right)^{2}} \mathbb{I}_{\left\{\vartheta_{j}+\frac{v_{j}}{T}<X_{s} \leq \vartheta_{j}+\frac{u_{j}}{T}\right\}} \mathrm{d} X_{s}\right. \\
\left.-\frac{1}{32} \int_{0}^{t} \frac{S_{j}\left(X_{s}\right)^{2}-S_{j+1}\left(X_{s}\right)^{2}}{\sigma\left(X_{s}\right)^{2}} \mathbb{I}_{\left\{\vartheta_{j}+\frac{v_{j}}{T}<X_{s} \leq \vartheta_{j}+\frac{u_{j}}{T}\right\}} \mathrm{d} s\right\}
\end{aligned}
$$

by Itô formula admits the representation (under measure $\mathbf{P}_{\boldsymbol{\vartheta}}^{(T)}$ )

$$
\begin{aligned}
V_{j, T}=1 & +\frac{1}{16} \int_{0}^{T} V_{j, t} \frac{S_{j}\left(X_{s}\right)-S_{j+1}\left(X_{s}\right)}{\sigma\left(X_{s}\right)} \mathbb{I}_{\left\{\vartheta_{j}+\frac{v_{j}}{T}<X_{s} \leq \vartheta_{j}+\frac{u_{j}}{T}\right\}} \mathrm{d} W_{t} \\
& -\frac{15}{512} \int_{0}^{T} V_{j, t}\left(\frac{S_{j}\left(X_{s}\right)-S_{j+1}\left(X_{s}\right)}{\sigma\left(X_{s}\right)}\right)^{2} \mathbb{I}_{\left\{\vartheta_{j}+\frac{v_{j}}{T}<X_{s} \leq \vartheta_{j}+\frac{u_{j}}{T}\right\}} \mathrm{d} t,
\end{aligned}
$$

Remind that

$$
\mathbb{I}_{\left\{\vartheta_{j}+\frac{v_{j}}{T}<x \leq \vartheta_{j}+\frac{u_{j}}{T}\right\}} \sum_{l=1}^{k+1} S_{l}(x) \mathbb{I}_{\left\{\vartheta_{l-1}<x \leq \vartheta_{l}\right\}}=S_{j+1}(x) \mathbb{I}_{\left\{\vartheta_{j}+\frac{v_{j}}{T}<x \leq \vartheta_{j}+\frac{u_{j}}{T}\right\}} .
$$

Therefore we can write

$$
\begin{aligned}
& \mathbf{E}_{\vartheta}\left|Z_{j, T}^{1 / 16}\left(u_{j}\right)-Z_{j, T}^{1 / 16}\left(v_{j}\right)\right|^{4}=\mathbf{E}_{\vartheta} Z_{j, T}^{1 / 4}\left(v_{j}\right)\left|1-V_{j, T}\right|^{4} \\
& \quad \leq\left(\mathbf{E}_{\vartheta} Z_{j, T}^{1 / 2}\left(v_{j}\right)\right)^{1 / 2}\left(\mathbf{E}_{\vartheta}\left|1-V_{j, T}\right|^{8}\right)^{1 / 2} \leq\left(\mathbf{E}_{\vartheta}\left|1-V_{j, T}\right|^{8}\right)^{1 / 2}
\end{aligned}
$$

because $\mathbf{E}_{\vartheta} Z_{j, T}^{1 / 2}\left(v_{j}\right) \leq 1$. Further

$$
\begin{gathered}
\mathbf{E}_{\vartheta}\left|1-V_{j, T}\right|^{8} \leq C_{1} \mathbf{E}_{\vartheta}\left(\int_{0}^{T} V_{j, t} g_{j}\left(X_{t}\right)^{2} \mathbb{I}_{\left\{\vartheta_{j}+\frac{v_{j}}{T}<X_{s} \leq \vartheta_{j}+\frac{u_{j}}{T}\right\}} \mathrm{d} t\right)^{8} \\
+C_{2} \mathbf{E}_{\vartheta}\left(\int_{0}^{T} V_{j, t} g_{j}\left(X_{t}\right) \mathbb{1}_{\left\{\vartheta_{j}+\frac{v_{j}}{T}<X_{s} \leq \vartheta_{j}+\frac{u_{j}}{T}\right\}} \mathrm{d} W_{t}\right)^{8} .
\end{gathered}
$$


For the last (stochastic) integral we have the estimates

$$
\begin{aligned}
\mathbf{E}_{\vartheta}\left(\int_{0}^{T} V_{j, t} g_{j}\left(X_{t}\right) \mathbb{I}_{\left\{\vartheta_{j}+\frac{v_{j}}{T}<X_{s} \leq \vartheta_{j}+\frac{u_{j}}{T}\right\}} \mathrm{d} W_{t}\right)^{8} \\
\quad \leq C \mathbf{E}_{\vartheta}\left(\int_{0}^{T} V_{j, t}^{2} g_{j}\left(X_{t}\right)^{2} \mathbb{I}_{\left\{\vartheta_{j}+\frac{v_{j}}{T}<X_{s} \leq \vartheta_{j}+\frac{u_{j}}{T}\right\}} \mathrm{d} t\right)^{4} \\
\quad \leq C \mathbf{E}_{\vartheta} \sup _{0 \leq t \leq T} V_{j, t}^{8}\left(\int_{0}^{T} g_{j}\left(X_{t}\right)^{2} \mathbb{I}_{\left\{\vartheta_{j}+\frac{v_{j}}{T}<X_{s} \leq \vartheta_{j}+\frac{u_{j}}{T}\right\}} \mathrm{d} t\right)^{4} \\
\leq C\left(\mathbf{E}_{\vartheta} \sup _{0 \leq t \leq T} V_{j, t}^{16}\right)^{1 / 2} \\
\quad\left(\mathbf{E}_{\vartheta}\left(\int_{0}^{T} g_{j}\left(X_{t}\right)^{2} \mathbb{I}_{\left\{\vartheta_{j}+\frac{v_{j}}{T}<X_{s} \leq \vartheta_{j}+\frac{u_{j}}{T}\right\}} \mathrm{d} t\right)^{8}\right)^{1 / 2} .
\end{aligned}
$$

Remind that $V_{t}^{16}$ is martingale and $\mathbf{E}_{\vartheta} V_{T}^{16}=1$. Using once more the local time estimator of the density we write

$$
\int_{0}^{T} g_{j}\left(X_{t}\right)^{2} \mathbb{I}_{\left\{\vartheta_{j}+\frac{v_{j}}{T}<X_{s} \leq \vartheta_{j}+\frac{u_{j}}{T}\right\}} \mathrm{d} t=T \int_{\vartheta_{j}+\frac{v_{j}}{T}}^{\vartheta_{j}+\frac{u_{j}}{T}} g_{j}(x)^{2} f_{T}^{\circ}(x) \mathrm{d} x .
$$

Hence

$$
\begin{aligned}
& \mathbf{E}_{\vartheta}\left(\int_{0}^{T} g_{j}\left(X_{t}\right)^{2} \mathbb{I}_{\left\{\vartheta_{j}+\frac{v_{j}}{T}<X_{s} \leq \vartheta_{j}+\frac{u_{j}}{T}\right\}} \mathrm{d} t\right)^{8} \\
& \quad \leq\left(u_{j}-v_{j}\right)^{7} T \int_{\vartheta_{j}+\frac{v_{j}}{T}}^{\vartheta_{j}+\frac{u_{j}}{T}} g_{j}(x)^{16} \mathbf{E}_{\vartheta} f_{T}^{\circ}(x)^{8} \mathrm{~d} x \leq C\left(u_{j}-v_{j}\right)^{8} .
\end{aligned}
$$

The expectation $\mathbf{E}_{\vartheta} f_{T}^{\circ}(x)^{8}$ due to the estimate (24) is a bounded function. For the first integral in (26) the similar calculations yield the estimate

$$
\mathbf{E}_{\vartheta}\left(\int_{0}^{T} V_{j, t} g_{j}\left(X_{t}\right)^{2} \mathbb{I}_{\left\{\vartheta_{j}+\frac{v_{j}}{T}<X_{s} \leq \vartheta_{j}+\frac{u_{j}}{T}\right\}} \mathrm{d} t\right)^{8} \leq C\left(u_{j}-v_{j}\right)^{8} .
$$

Therefore, for $\left|u_{j}\right| \leq R,\left|v_{j}\right| \leq R$

$$
\begin{aligned}
\mathbf{E}_{\vartheta}\left|Z_{j, T}^{1 / 16}\left(u_{j}\right)-Z_{j, T}^{1 / 16}\left(v_{j}\right)\right|^{8} & \leq C\left(u_{j}-v_{j}\right)^{2}+\left(u_{j}-v_{j}\right)^{4} \\
& \leq C\left(1+R^{2}\right)\left|u_{j}-v_{j}\right|^{2}
\end{aligned}
$$


To verify condition $\mathbf{C}$ we follow the proof of the Lemmas 3.29 and 2.11 in [13. By condition (18) we have

$$
\begin{aligned}
& \mathbf{E}_{\vartheta} \sum_{j=1}^{k} \int_{0}^{T}\left[h_{j}\left(X_{t}\right)-h_{j+1}\left(X_{t}\right)\right]^{2} \mathbb{1}_{\left\{\vartheta_{j}<X_{t} \leq \vartheta_{j}+\delta_{j}\right\}} \mathrm{d} t \\
& =T \sum_{j=1}^{k} \int_{\vartheta_{j}}^{\vartheta_{j}+\delta_{j}}\left[h_{j}(x)-h_{j+1}(x)\right]^{2} f(\boldsymbol{\vartheta}, x) \mathrm{d} x \\
& =T \sum_{j=1}^{k} \kappa_{j} \delta_{j}(1+o(1)) \geq \kappa T|\boldsymbol{\delta}|
\end{aligned}
$$

with some positive constants $\kappa, \kappa_{j}$. Here $\boldsymbol{\delta}=\left(\delta_{1}, \ldots, \delta_{k}\right)$ and we suppose for simplicity that all $\delta_{j}>0$. Hence the inequality (23) follows from the mentioned above lemmas.

The properties of BE follow from the Theorem 1.10.2 in 7] because the conditions A, (25) and (23) are sufficient for this theorem.

For the MLE we do not apply directly the Theorem 1.10.1 in [7] because it requires in condition $\mathbf{B}$ that $d>k$. We follow the modification of this theorem discussed in the proof of the Proposition 2.40 in [13]. Let us consider the vector of likelihood $\operatorname{ratios} \boldsymbol{Y}(\boldsymbol{u})_{T}=\left(Z_{1, T}^{1 / 4}\left(u_{1}\right), \ldots, Z_{k, T}^{1 / 4}\left(u_{1}\right)\right)$. For the components $Z_{j, T}^{1 / 4}\left(u_{j}\right), j=1, \ldots, k$ we have the joint convergence of its dimensional distributions to the distribution of the limit random field $\boldsymbol{Y}(\boldsymbol{u})=\left(Z_{1}^{1 / 4}\left(u_{1}\right), \ldots, Z_{k}^{1 / 4}\left(u_{1}\right)\right)$ with independent components and the conditions $\mathbf{B}$ and $\mathbf{C}$. Therefore we have the tightness of the corresponding vector of measures and for each component we have the large deviations estimates: for any $L>0$ and $N>0$ there exists $C_{N}>0$ such that

$$
\mathbf{P}_{\vartheta}^{(T)}\left\{\sup _{\left|u_{j}\right|>L} Z_{j, T}^{1 / 4}\left(u_{j}\right) \geq \frac{1}{L^{N}}\right\} \leq \frac{C_{N}}{L^{N}} .
$$

These estimates and the factorization of the likelihood ratio (17) allows us to finish the proof of the properties of MLE mentioned in Theorem 1. Note that the MLE $\hat{\vartheta}_{j, T}$ can be written as

$$
\hat{\vartheta}_{j, T}=\operatorname{argmax}_{\theta_{j} \in \Theta_{j}} L_{j}^{1 / 4}\left(\theta_{j}, X^{T}\right)
$$

too. 
To prove the Proposition 2 we consider the normalized likelihood ratio (we take $u>0$ )

$$
\begin{aligned}
& \ln Z_{T}(v, w, u)=\ln \frac{L\left(\vartheta_{1}+\frac{v}{\sqrt{T}}, \vartheta_{2}+\frac{w}{\sqrt{T}}, \vartheta_{3}+\frac{u}{T}, X^{T}\right)}{L\left(\vartheta_{1}, \vartheta_{2}, \vartheta_{3}, X^{T}\right)} \\
&=-\frac{v}{\sigma \sqrt{T}} \int_{0}^{T} X_{t} \mathbb{I}_{\left\{X_{t}<\vartheta_{3}\right\}} \mathrm{d} W_{t}-\frac{w}{\sigma \sqrt{T}} \int_{0}^{T} X_{t} \mathbb{I}_{\left\{X_{t} \geq \vartheta_{3}\right\}} \mathrm{d} W_{t} \\
&+\left(\vartheta_{2}-\vartheta_{1}+\frac{w-v}{\sqrt{T}}\right) \frac{1}{\sigma} \int_{0}^{T} X_{t} \mathbb{I}_{\left\{\vartheta_{3}<X_{t} \leq \vartheta_{3}+\frac{u}{\sqrt{T}}\right\}} \mathrm{d} W_{t} \\
& \quad-\frac{1}{\sigma^{2}} \int_{0}^{T}\left[-\frac{v}{\sqrt{T}} \mathbb{I}_{\left\{X_{t}<\vartheta_{3}\right\}}-\frac{w}{\sqrt{T}} \mathbb{I}_{\left\{X_{t} \geq \vartheta_{3}\right\}}\right. \\
&\left.+\left(\vartheta_{2}-\vartheta_{1}+\frac{w-v}{\sqrt{T}}\right) 1_{\left\{\vartheta_{3}<X_{t} \leq \vartheta_{3}+\frac{u}{\sqrt{T}}\right\}}\right]^{2} X_{t}^{2} \mathrm{~d} t \\
& \equiv v \Delta_{1, T}+w \Delta_{2, T}+\left(\frac{\vartheta_{2}-\vartheta_{1}}{\sigma}+\frac{w-v}{\sigma \sqrt{T}}\right) \Delta_{3, T}(u)-\frac{1}{2} J_{T},
\end{aligned}
$$

where the last equality introduce the notation for these integrals. For the last integral we can write

$$
\begin{aligned}
J_{T}= & \frac{v^{2}}{\sigma^{2} T} \int_{0}^{T} X_{t}^{2} \mathbb{I}_{\left\{X_{t}<\vartheta_{3}\right\}} \mathrm{d} t+\frac{w^{2}}{\sigma^{2} T} \int_{0}^{T} X_{t}^{2} \mathbb{I}_{\left\{X_{t} \geq \vartheta_{3}\right\}} \mathrm{d} t \\
& +\frac{\left(\vartheta_{2}-\vartheta_{1}\right)^{2}}{\sigma^{2}} \int_{0}^{T} X_{t}^{2} \mathbb{I}_{\left\{\vartheta_{3}<X_{t} \leq \vartheta_{3}+\frac{u}{T}\right\}} \mathrm{d} t+o(1) .
\end{aligned}
$$

For the first two integrals by the law of large numbers we have

$$
\begin{aligned}
& \frac{1}{T} \int_{0}^{T} X_{t}^{2} \mathbb{I}_{\left\{X_{t}<\vartheta_{3}\right\}} \mathrm{d} t \longrightarrow \mathbf{E}_{\vartheta} \xi^{2} \mathbb{I}_{\left\{\xi<\vartheta_{3}\right\}}, \\
& \frac{1}{T} \int_{0}^{T} X_{t}^{2} \mathbb{I}_{\left\{X_{t} \geq \vartheta_{3}\right\}} \mathrm{d} t \longrightarrow \mathbf{E}_{\vartheta} \xi^{2} \mathbb{I}_{\left\{\xi \geq \vartheta_{3}\right\}},
\end{aligned}
$$

and for the last one using the local time estimator of the density we obtain

$$
\begin{aligned}
& \int_{0}^{T} X_{t}^{2} \mathbb{I}_{\left\{\vartheta_{3}<X_{t} \leq \vartheta_{3}+\frac{u}{T}\right\}} \mathrm{d} t=T \int_{\vartheta_{3}}^{\vartheta_{3}+\frac{u}{T}} x^{2} f_{T}^{\circ}(x) \mathrm{d} x=T \int_{\vartheta_{3}}^{\vartheta_{3}+\frac{u}{T}} x^{2} f(\boldsymbol{\vartheta}, x) \mathrm{d} x \\
& +T \int_{\vartheta_{3}}^{\vartheta_{3}+\frac{u}{T}} x^{2}\left(f_{T}^{\circ}(x)-f(\boldsymbol{\vartheta}, x)\right) \mathrm{d} x=u \vartheta_{3}^{2} f\left(\boldsymbol{\vartheta}, \vartheta_{3}\right)+o(1),
\end{aligned}
$$

where in $o(1)$ we used once more the estimate (24). Therefore

$$
J_{T} \longrightarrow \frac{v^{2}}{\sigma^{2}} \mathbf{E}_{\boldsymbol{\vartheta}} \xi^{2} \mathbb{I}_{\left\{\xi \leq \vartheta_{3}\right\}}+\frac{w^{2}}{\sigma^{2}} \mathbf{E}_{\vartheta} \xi^{2} \mathbb{I}_{\left\{\xi \geq \vartheta_{3}\right\}}+u \frac{\left(\vartheta_{2}-\vartheta_{1}\right)^{2} \vartheta_{3}^{2}}{\sigma^{2}} f\left(\boldsymbol{\vartheta}, \vartheta_{3}\right) .
$$


For the stochastic integrals $\Delta_{1, T}$ and $\Delta_{2, T}$ from (29), (30) and by the central limit theorem we have the convergence

$$
\begin{array}{ll}
\Delta_{1, T} \Longrightarrow \zeta_{1} \sim \mathcal{N}\left(0, \mathrm{I}_{1}\right), & \mathrm{I}_{1}=\frac{1}{\sigma^{2}} \mathbf{E}_{\vartheta} \xi^{2} \mathbb{I}_{\left\{\xi \leq \vartheta_{3}\right\}} \\
\Delta_{2, T} \Longrightarrow \zeta_{2} \sim \mathcal{N}\left(0, \mathrm{I}_{2}\right), & \mathrm{I}_{2}=\frac{1}{\sigma^{2}} \mathbf{E}_{\vartheta} \xi^{2} \mathbb{I}_{\left\{\xi \geq \vartheta_{3}\right\}},
\end{array}
$$

where the random variables $\zeta_{1}$ and $\zeta_{2}$ are independent.

Let us consider $\Delta_{T}=\lambda_{1} \Delta_{3, T}\left(u_{1}\right)+\lambda_{2} \Delta_{3, T}\left(u_{2}\right)$. We have

$$
\Delta_{T}=\int_{0}^{T}\left[\lambda_{1} X_{t} \mathbb{I}_{\left\{\vartheta_{3}<X_{t} \leq \vartheta_{3}+\frac{u_{1}}{T}\right\}}+\lambda_{2} X_{t} \mathbb{I}_{\left\{\vartheta_{3}<X_{t} \leq \vartheta_{3}+\frac{u_{2}}{T}\right\}}\right] \mathrm{d} W_{t} .
$$

Note that

$$
\begin{aligned}
\int_{0}^{T} & {\left[\lambda_{1} X_{t} \mathbb{I}_{\left\{\vartheta_{3}<X_{t} \leq \vartheta_{3}+\frac{u_{1}}{T}\right\}}+\lambda_{2} X_{t} \mathbb{I}_{\left\{\vartheta_{3}<X_{t} \leq \vartheta_{3}+\frac{u_{2}}{T}\right\}}\right]^{2} \mathrm{~d} t } \\
= & \lambda_{1}^{2} \int_{0}^{T} X_{t}^{2} \mathbb{I}_{\left\{\vartheta_{3}<X_{t} \leq \vartheta_{3}+\frac{u_{1}}{T}\right\}} \mathrm{d} t+\lambda_{2}^{2} \int_{0}^{T} X_{t}^{2} \mathbb{I}_{\left\{\vartheta_{3}<X_{t} \leq \vartheta_{3}+\frac{u_{2}}{T}\right\}} \mathrm{d} t \\
& +2 \lambda_{1} \lambda_{2} \int_{0}^{T} X_{t}^{2} \mathbb{I}_{\left\{\vartheta_{3}<X_{t} \leq \vartheta_{3}+\frac{u_{1} \wedge u_{2}}{T}\right\}} \mathrm{d} t \\
\longrightarrow & {\left[u_{1} \lambda_{1}^{2}+u_{2} \lambda_{2}^{2}+2 \lambda_{1} \lambda_{2}\left(u_{1} \wedge u_{2}\right)\right] \vartheta_{3}^{2} f\left(\boldsymbol{\vartheta}, \vartheta_{3}\right) \equiv d^{2} . }
\end{aligned}
$$

Hence $\Delta_{T}$ is asymptotically normal $\Delta_{T} \Rightarrow \Delta$ with the limit variance $d^{2}$. Remind that the same variance has the random variable

$$
\Delta=\lambda_{1} \vartheta_{3} \sqrt{f\left(\boldsymbol{\vartheta}, \vartheta_{3}\right)} W\left(u_{1}\right)+\lambda_{2} \vartheta_{3} \sqrt{f\left(\boldsymbol{\vartheta}, \vartheta_{3}\right)} W\left(u_{2}\right),
$$

where $W(\cdot)$ is a Wiener process. Therefore we have the convergence of the finite dimensional distributions of $\Delta_{3, T}(u)$ to the finite dimensional distributions of the process $\vartheta_{3} \sqrt{f\left(\boldsymbol{\vartheta}, \vartheta_{3}\right)} W(u)$ :

$$
\begin{aligned}
& \left(\Delta_{3, T}\left(u_{1}\right), \ldots, \Delta_{3, T}\left(u_{k}\right)\right) \\
& \quad \Longrightarrow\left(\vartheta_{3} \sqrt{f\left(\boldsymbol{\vartheta}, \vartheta_{3}\right)} W\left(u_{1}\right), \ldots, \vartheta_{3} \sqrt{f\left(\boldsymbol{\vartheta}, \vartheta_{3}\right)} W\left(u_{k}\right)\right)
\end{aligned}
$$

This convergence together with (31) and (32) allows to write the likelihood ratio random field as

$$
\begin{aligned}
Z_{T}(v, w, u)=\exp & \left\{v \Delta_{1, T}-\frac{v^{2}}{2} \mathrm{I}_{1}+w \Delta_{2, T}-\frac{w^{2}}{2} \mathrm{I}_{2}\right. \\
+ & \left.\left(\frac{\vartheta_{2}-\vartheta_{1}}{\sigma}\right) \Delta_{3, T}(u)-\frac{|u|}{2} \gamma(\boldsymbol{\vartheta})^{2}+o(1)\right\}
\end{aligned}
$$


where $\Delta_{1, T}$ and $\Delta_{2, T}$ are asymptotically normal, and

$$
\gamma(\boldsymbol{\vartheta})^{2}=\frac{\left(\vartheta_{2}-\vartheta_{1}\right)^{2} \vartheta_{3}^{2}}{\sigma^{2}} f\left(\boldsymbol{\vartheta}, \vartheta_{3}\right) \equiv \gamma^{2}
$$

Therefore we have the convergence of the finite dimensional distributions of $Z_{T}(v, w, u)$ to that of the random function

$$
Z(v, w, u)=e^{v \zeta_{1}-\frac{v^{2}}{2} \mathrm{I}_{1}} e^{w \zeta_{2}-\frac{w^{2}}{2} \mathrm{I}_{2}} e^{\gamma W(u)-\frac{|u|}{2} \gamma^{2}}, \quad v, w, u \in \mathscr{R}^{3}
$$

where $\zeta_{1}, \zeta_{2}$ and $W(\cdot)$ are independent.

To check the condition $\mathbf{B}$ in the case of Bayesian estimation we following (25) $)$ write $\left(u_{2}>u_{1}>0\right)$

$$
\begin{aligned}
\mathbf{E}_{\vartheta} \mid & Z_{T}^{1 / 2}\left(v_{1}, w_{1}, u_{1}\right)-\left.Z_{T}^{1 / 2}\left(v_{2}, w_{2}, u_{2}\right)\right|^{2} \\
\leq & \frac{1}{4 \sigma^{2}} \mathbf{E}_{*} \int_{0}^{T}\left[\frac{\left(v_{1}-v_{2}\right) \mathbb{1}_{\left\{X_{t}<\vartheta_{3}\right\}}}{\sqrt{T}}+\frac{\left(w_{1}-w_{2}\right) \mathbb{I}_{\left\{X_{t} \geq \vartheta_{3}\right\}}}{\sqrt{T}}\right. \\
& \left.+\left(\vartheta_{1}-\vartheta_{2}+\frac{v_{2}-v_{1}-w_{2}+w_{1}}{\sqrt{T}}\right) \mathbb{I}_{\left\{\vartheta_{3}+\frac{u_{1}}{T}<X_{t}<\vartheta_{3}+\frac{u_{2}}{T}\right\}}\right]^{2} X_{t}^{2} \mathrm{~d} t \\
\leq & C_{1}\left(v_{1}-v_{2}\right)^{2}+C_{2}\left(w_{1}-w_{2}\right)^{2}+C_{3}\left|u_{2}-u_{1}\right| .
\end{aligned}
$$

In the case of MLE this estimate is not sufficient because the condition $d>3$ is not fulfilled. We slightly modify the proof of (27). Let us denote $\boldsymbol{u}=(v, w, u)$ and put

$$
V_{T}=\left(\frac{Z_{T}\left(v_{2}, w_{2}, u_{2}\right)}{Z_{T}\left(v_{1}, w_{1}, u_{1}\right)}\right)^{\frac{1}{32}}
$$

Then

$$
\begin{gathered}
\mathbf{E}_{\vartheta}\left|Z_{T}^{\frac{1}{32}}\left(v_{1}, w_{1}, u_{1}\right)-Z_{T}^{\frac{1}{32}}\left(v_{2}, w_{2}, u_{2}\right)\right|^{8}=\mathbf{E}_{\vartheta} Z_{T}^{\frac{1}{4}}\left(v_{2}, w_{2}, u_{2}\right)\left|1-V_{T}\right|^{8} \\
\quad \leq\left(\mathbf{E}_{\vartheta} Z_{T}^{\frac{1}{2}}\left(v_{2}, w_{2}, u_{2}\right)\right)^{\frac{1}{2}}\left(\mathbf{E}_{\vartheta}\left|1-V_{T}\right|^{16}\right)^{\frac{1}{2}} \leq\left(\mathbf{E}_{\vartheta}\left|1-V_{T}\right|^{16}\right)^{\frac{1}{2}}
\end{gathered}
$$

The process $V_{t}, 0 \leq t \leq T$ by Itô formula admits the representation

$$
V_{T}=1-a \int_{0}^{T} V_{t}\left(\Delta S\left(X_{t}\right)\right)^{2} \mathrm{~d} t+b \int_{0}^{T} V_{t}\left(\Delta S\left(X_{t}\right)\right) \mathrm{d} W_{t}
$$

with corresponding constants $a>0$ and $b>0$ and $\Delta S\left(X_{t}\right) \equiv \Delta S_{t}$ is the difference of two trend coefficients. Hence

$$
\begin{aligned}
\mathbf{E}_{\vartheta}\left|1-V_{T}\right|^{16} & \leq A \mathbf{E}_{\vartheta}\left(\int_{0}^{T} V_{t}\left(\Delta S_{t}\right)^{2} \mathrm{~d} t\right)^{16}+B \mathbf{E}_{\vartheta}\left(\int_{0}^{T} V_{t}\left(\Delta S_{t}\right) \mathrm{d} t\right)^{16} \\
& \leq A \mathbf{E}_{\vartheta}\left(\int_{0}^{T} V_{t}\left(\Delta S_{t}\right)^{2} \mathrm{~d} t\right)^{16}+C \mathbf{E}_{\vartheta}\left(\int_{0}^{T} V_{t}^{2}\left(\Delta S_{t}\right)^{2} \mathrm{~d} t\right)^{8} .
\end{aligned}
$$


Further

$$
\begin{aligned}
& \mathbf{E}_{\vartheta}\left(\int_{0}^{T} V_{t}^{2}\left(\Delta S_{t}\right)^{2} \mathrm{~d} t\right)^{8} \leq \mathbf{E}_{\vartheta} \sup _{0 \leq t \leq T} V_{t}^{16}\left(\int_{0}^{T}\left(\Delta S_{t}\right)^{2} \mathrm{~d} t\right)^{8} \\
& \leq\left(\mathbf{E}_{\vartheta} \sup _{0 \leq t \leq T} V_{t}^{24}\right)^{\frac{2}{3}}\left(\mathbf{E}_{\vartheta}\left(\int_{0}^{T}\left(\Delta S_{t}\right)^{2} \mathrm{~d} t\right)^{24}\right)^{\frac{1}{3}} \\
& \quad \leq\left(\mathbf{E}_{\vartheta}\left(\int_{0}^{T}\left(\Delta S_{t}\right)^{2} \mathrm{~d} t\right)^{24}\right)^{\frac{1}{3}}
\end{aligned}
$$

because $\mathbf{E}_{\vartheta} \sup _{0 \leq t \leq T} V_{t}^{24} \leq 1$. Now with the help of (28) we can write

$$
\begin{aligned}
& \mathbf{E}_{\vartheta}\left(\int_{0}^{T}\left(\Delta S\left(X_{t}\right)\right)^{2} \mathrm{~d} t\right)^{24}=\mathbf{E}_{\vartheta}\left(T \int_{-\infty}^{\infty}(\Delta S(x))^{2} f_{T}^{\circ}(x) \mathrm{d} x\right)^{24} \\
& \quad \leq C_{1}\left(v_{2}-v_{1}\right)^{48}+C_{2}\left(w_{2}-w_{1}\right)^{48}+C_{3}\left(u_{2}-u_{1}\right)^{24}
\end{aligned}
$$

After substitution of these estimates we obtain

$$
\begin{aligned}
\mathbf{E}_{\vartheta} \mid Z_{T}^{\frac{1}{32}} & \left(v_{1}, w_{1}, u_{1}\right)-\left.Z_{T}^{\frac{1}{32}}\left(v_{2}, w_{2}, u_{2}\right)\right|^{8} \\
& \leq A\left|v_{2}-v_{1}\right|^{8}+B\left|w_{2}-w_{1}\right|^{8}+C\left|u_{2}-u_{1}\right|^{4}
\end{aligned}
$$

Therefore for the values $\left|v_{i}\right|+\left|w_{i}\right|+\left|u_{i}\right| \leq R$ we have

$$
\begin{aligned}
\mathbf{E}_{\vartheta} \mid Z_{T}^{\frac{1}{32}} & \left(v_{1}, w_{1}, u_{1}\right)-\left.Z_{T}^{\frac{1}{32}}\left(v_{2}, w_{2}, u_{2}\right)\right|^{8} \\
& \leq C\left(1+R^{4}\right)\left(\left|v_{2}-v_{1}\right|^{4}+\left|w_{2}-w_{1}\right|^{4}+\left|u_{2}-u_{1}\right|^{4}\right)
\end{aligned}
$$

Hence the condition $\mathbf{B}$ is fulfilled with $m=4$ and $d=4>3$ for the random field $Y_{T}(v, w, u)=Z_{T}^{\frac{1}{4}}(v, w, u)$.

To verify the condition $\mathbf{C}$ we follow the proof of Lemma 2.11 in [13]. We write $(u>0)$

$$
\begin{aligned}
\mathbf{E}_{\vartheta} J_{T}= & \frac{v^{2}}{\sigma^{2} T} \int_{0}^{T} \mathbf{E}_{\vartheta} X_{t}^{2} \mathbb{I}_{\left\{X_{t}<\vartheta_{3}\right\}} \mathrm{d} t+\frac{w^{2}}{\sigma^{2} T} \int_{0}^{T} \mathbf{E}_{\vartheta} X_{t}^{2} \mathbb{I}_{\left\{X_{t} \geq \vartheta_{3}\right\}} \mathrm{d} t \\
& +\left(\frac{\vartheta_{2}-\vartheta_{1}}{\sigma}+\frac{v-w}{\sigma \sqrt{T}}\right)^{2} \int_{0}^{T} \mathbf{E}_{\vartheta} X_{t}^{2} \mathbb{I}_{\left\{\vartheta_{3}<X_{t} \leq \vartheta_{3}+\frac{u}{T}\right\}} \mathrm{d} t \\
& +2 \frac{w}{\sqrt{T}}\left(\frac{\vartheta_{2}-\vartheta_{1}}{\sigma}+\frac{v-w}{\sigma \sqrt{T}}\right) \int_{0}^{T} \mathbf{E}_{\vartheta} X_{t}^{2} \mathbb{I}_{\left\{\vartheta_{3}<X_{t} \leq \vartheta_{3}+\frac{u}{T}\right\}} \mathrm{d} t .
\end{aligned}
$$


Note that

$$
0<\kappa \equiv \frac{\alpha_{2}-\beta_{1}}{\sigma}<\frac{1}{\sigma}\left|\vartheta_{2}+\frac{w}{\sqrt{T}}-\left(\vartheta_{1}+\frac{v}{\sqrt{T}}\right)\right|<\frac{\beta_{2}-\alpha_{1}}{\sigma} \equiv K
$$

Hence

$$
\begin{aligned}
\mathbf{E}_{\vartheta} J_{T} \geq \frac{v^{2}}{\sigma^{2}} \mathbf{E}_{\vartheta} \xi^{2} \mathbb{I}_{\left\{\xi<\vartheta_{3}\right\}}+\frac{w^{2}}{\sigma^{2}} \mathbf{E}_{\vartheta} \xi^{2} \mathbb{I}_{\left\{\xi \geq \vartheta_{3}\right\}}+\kappa^{2} T \int_{\vartheta_{3}}^{\vartheta_{3}+\frac{u}{T}} x^{2} f(\boldsymbol{\vartheta}, x) \mathrm{d} x \\
-2 \frac{|w|}{\sqrt{T}} K T \int_{\vartheta_{3}}^{\vartheta_{3}+\frac{u}{T}} x^{2} f(\boldsymbol{\vartheta}, x) \mathrm{d} x .
\end{aligned}
$$

Let us put $\delta=\kappa^{2} / 4 K$, then for $\frac{|v|}{\sqrt{T}}+\frac{|w|}{\sqrt{T}}+\frac{|u|}{T} \leq \delta$ we have

$$
\mathbf{E}_{\vartheta} J_{T} \geq v^{2} \mathrm{I}_{1}+w^{2} \mathrm{I}_{2}+|u| \frac{\kappa^{2}}{2} \alpha_{3}^{2} \inf _{\alpha_{3}<x \leq \beta_{3}} f(\boldsymbol{\vartheta}, x)
$$

and for the vector $\boldsymbol{h}=\left(h_{1}, h_{2}, h_{3}\right)$ with $h_{1}=\frac{v}{\sqrt{T}}, h_{2}=\frac{w}{\sqrt{T}}, h_{3}=\frac{u}{T}$, and $\|\boldsymbol{h}\| \geq \delta$ we can write

$$
\begin{aligned}
\frac{\mathbf{E}_{\boldsymbol{\vartheta}} J_{T}}{T}= & h_{1}^{2} \int_{-\infty}^{\vartheta_{3}} x^{2} f(\boldsymbol{\vartheta}, x) \mathrm{d} x+h_{2}^{2} \int_{\vartheta_{3}+h_{3}}^{\infty} x^{2} f(\boldsymbol{\vartheta}, x) \mathrm{d} x \\
& +\left(\vartheta_{1}-\vartheta_{2}+h_{1}\right)^{2} \int_{\vartheta_{3}}^{\vartheta_{3}+h_{3}} x^{2} f(\boldsymbol{\vartheta}, x) \mathrm{d} x \\
\geq & h_{1}^{2} \int_{-\infty}^{\vartheta_{3}} x^{2} f(\boldsymbol{\vartheta}, x) \mathrm{d} x+h_{2}^{2} \int_{\beta_{3}}^{\infty} x^{2} f(\boldsymbol{\vartheta}, x) \mathrm{d} x \\
& +\left(\alpha_{2}-\beta_{1}\right)^{2} \int_{\vartheta_{3}}^{\vartheta_{3}+h_{3}} x^{2} f(\boldsymbol{\vartheta}, x) \mathrm{d} x>\kappa_{1}>0 .
\end{aligned}
$$

Here we used the representation

$$
\begin{array}{r}
-\vartheta_{1} \mathbb{I}_{\left\{x<\vartheta_{3}\right\}}-\vartheta_{2} \mathbb{I}_{\left\{x \geq \vartheta_{3}\right\}}+\left(\vartheta_{1}+h_{1}\right) \mathbb{I}_{\left\{x<\vartheta_{3}+h_{3}\right\}}-\left(\vartheta_{2}+h_{2}\right) \mathbb{I}_{\left\{x \geq \vartheta_{3}+h_{3}\right\}} \\
=h_{1} \mathbb{I}_{\left\{x<\vartheta_{3}\right\}}+h_{2} \mathbb{I}_{\left\{x \geq \vartheta_{3}+h_{3}\right\}}+\left(\vartheta_{1}-\vartheta_{2}+h_{1}\right) \mathbb{I}_{\left\{\vartheta_{3}<x \leq \vartheta_{3}+h_{3}\right\}} .
\end{array}
$$

Now having (35) and (36) we can follow the proof of Lemma 2.11 in 13 and obtain the estimate (23).

The properties of modified (simplified) estimators defined by the equalities (8) will be proved if we verify the law of large numbers (91). For any 
$\varepsilon>0$ using the consistency (17) we can write

$$
\begin{aligned}
\mathbf{P}_{\vartheta} & \left\{\left|\frac{1}{\sigma^{2} T} \int_{\sqrt{T}}^{T} X_{t}^{2} \mathbb{I}_{\left\{X_{t}<\hat{\vartheta}_{3, T}\right\}} \mathrm{d} t-\mathrm{I}_{1}\right|>\varepsilon\right\} \leq \mathbf{P}_{\vartheta}\left\{\left|\hat{\vartheta}_{3, T}-\vartheta_{3}\right| \geq T^{-b}\right\} \\
& +\mathbf{P}_{\vartheta}\left\{\left|\frac{1}{\sigma^{2} T} \int_{\sqrt{T}}^{T} X_{t}^{2} \mathbb{I}_{\left\{X_{t}<\hat{\vartheta}_{3, T}\right\}} \mathrm{d} t-\mathrm{I}_{1}\right|>\varepsilon,\left|\hat{\vartheta}_{3, T}-\vartheta_{3}\right|<T^{-b}\right\} \\
& \leq \mathbf{P}_{\vartheta}\left\{\sup _{\left|\theta-\vartheta_{3}\right|<T^{-b}}\left|\frac{1}{\sigma^{2} T} \int_{\sqrt{T}}^{T} X_{t}^{2} \mathbb{I}_{\left\{X_{t}<\theta\right\}} \mathrm{d} t-\mathrm{I}_{1}\right|>\varepsilon\right\}+o(1) .
\end{aligned}
$$

Further

$$
\begin{aligned}
& \sup _{\left|\theta-\vartheta_{3}\right|<T^{-b}}\left|\frac{1}{\sigma^{2} T} \int_{\sqrt{T}}^{T} X_{t}^{2} \mathbb{I}_{\left\{X_{t}<\theta\right\}} \mathrm{d} t-\mathrm{I}_{1}\right| \\
& \quad=\sup _{\left|\theta-\vartheta_{3}\right|<T^{-b}}\left|\frac{1}{\sigma^{2} T} \int_{0}^{T} X_{t}^{2} \mathbb{I}_{\left\{X_{t}<\theta\right\}} \mathrm{d} t-\mathrm{I}_{1}\right|+o(1) \\
& \quad=\sup _{\left|\theta-\vartheta_{3}\right|<T^{-b}}\left|\int_{-\infty}^{\theta} \frac{x^{2}}{\sigma^{2}} f_{T}^{\circ}(x) \mathrm{d} x-\int_{-\infty}^{\vartheta_{3}} \frac{x^{2}}{\sigma^{2}} f(\boldsymbol{\vartheta}, x) \mathrm{d} x\right|+o(1) \\
& \leq\left|\int_{-\infty}^{\vartheta_{3}} \frac{x^{2}}{\sigma^{2}}\left[f_{T}^{\circ}(x)-f(\boldsymbol{\vartheta}, x)\right] \mathrm{d} x\right|+\int_{\vartheta_{3}}^{\vartheta_{3}+T^{-b}} \frac{x^{2}}{\sigma^{2}} f_{T}^{\circ}(x) \mathrm{d} x+o(1) .
\end{aligned}
$$

To finish the proof we just mention, that

$$
\int_{-\infty}^{\vartheta_{3}} \frac{x^{2}}{\sigma^{2}}\left[f_{T}^{\circ}(x)-f(\boldsymbol{\vartheta}, x)\right] \mathrm{d} x \longrightarrow 0
$$

by the law of large numbers.

\section{Goodness of Fit Testing}

Remind two well known goodness of fit (GoF) tests of classical statistics [15]. If we observe $n$ i.i.d. random variables $\left(X_{1}, \ldots, X_{n}\right)=X^{n}$ with distribution function $F(x)$ and the basic hypothesis is simple

$$
\mathscr{H}_{0}, \quad F(x) \equiv F_{*}(x), \quad x \in \mathscr{R},
$$

then the Cramér-von Mises (C-vM) and Kolmogorov-Smirnov (K-S) statistics are

$$
\mathbb{W}_{n}^{2}=n \int\left[\hat{F}_{n}(x)-F_{*}(x)\right]^{2} \mathrm{~d} F_{*}(x), \quad \mathbb{D}_{n}=\sup _{x} \sqrt{n}\left|\hat{F}_{n}(x)-F_{*}(x)\right|
$$


respectively. Here $\hat{F}_{n}(x)$ is the empirical distribution function.

For continuous $F_{*}(x)$ under hypothesis $\mathscr{H}_{0}$ we have the convergence

$$
W_{n}^{2} \Longrightarrow \int_{0}^{1} W_{0}(s)^{2} \mathrm{~d} s, \quad \mathbb{D}_{n} \Longrightarrow \sup _{0 \leq s \leq 1}\left|W_{0}(s)\right|
$$

where $W_{0}(\cdot)$ is Brownian bridge. The limit distributions do not depend on the model $F_{*}(\cdot)$ (the tests are asymptotically distribution free) and this essentially simplifies the choice of the corresponding thresholds for the tests Cramér-von Mises and Kolmogorov-Smirnov. Note that the both tests are consistent against any fixed alternative.

Our goal is to discuss the possibility of the construction of asymptotically distribution free tests for the mentioned in this work threshold diffusion processes.

Suppose that the basic hypothesis is simple:

$$
\mathscr{H}_{0} \quad: \quad \text { the observed process } X^{T} \text { is } \operatorname{TOU}\left(\vartheta_{0}\right)
$$

i.e., the observations $X^{T}=\left(X_{t}, 0 \leq t \leq T\right)$ come from the equation

$$
\mathrm{d} X_{t}=-\rho_{1} X_{t} \mathbb{I}_{\left\{X_{t}<\vartheta_{0}\right\}} \mathrm{d} t-\rho_{2} X_{t} \mathbb{I}_{\left\{X_{t} \geq \vartheta_{0}\right\}} \mathrm{d} t+\sigma \mathrm{d} W_{t}, \quad 0 \leq t \leq T,
$$

with known $\vartheta_{0}$ and we have to test this hypothesis. We propose below some tests of C-vM and K-S types of asymptotic size $\alpha$.

Let us denote $g(x, \vartheta)=-\rho_{1} x \mathbb{1}_{\left\{x<\vartheta_{0}\right\}}-\rho_{2} x \mathbb{1}_{\left\{x \geq \vartheta_{0}\right\}}$ and following [3] introduce the statistics

$$
\mathbb{W}_{T}^{2}=\frac{1}{\sigma^{2} T^{2}} \int_{0}^{T}\left[X_{t}-X_{0}-\int_{0}^{t} g\left(X_{s}, \vartheta_{0}\right) \mathrm{d} s\right]^{2} \mathrm{~d} t,
$$

and

$$
\mathbb{D}_{T}=\frac{1}{\sigma \sqrt{T}} \sup _{0 \leq t \leq T}\left|X_{t}-X_{0}-\int_{0}^{t} g\left(X_{s}, \vartheta_{0}\right) \mathrm{d} s\right|
$$

It is easy to see that under $\mathscr{H}_{0}$ (= in distribution)

$$
\mathbb{W}_{T}^{2}=\int_{0}^{1} W(s)^{2} \mathrm{~d} s, \quad \mathbb{D}_{T}=\sup _{0 \leq s \leq 1}|W(s)|,
$$

where $W(\cdot)$ is Wiener process. Hence the tests

$$
\psi_{T}\left(X^{T}\right)=\mathbb{I}_{\left\{\mathbb{W}_{T}^{2}>c_{\alpha}\right\}}, \quad \phi_{T}\left(X^{T}\right)=\mathbb{I}_{\left\{\mathbb{D}_{T}>d_{\alpha}\right\}}
$$


are distribution free. Here the thresholds $c_{\alpha}, d_{\alpha}$ are solutions of the equations

$$
\mathbf{P}\left\{\int_{0}^{1} W(s)^{2} \mathrm{~d} s>c_{\alpha}\right\}=\alpha, \quad \mathbf{P}\left\{\sup _{0 \leq s \leq 1}|W(s)|>d_{\alpha}\right\}=\alpha .
$$

The both tests are consistent against any fixed alternative..

Suppose now that the basic hypothesis is composite:

$$
\mathscr{H}_{0} \quad \text { : } \quad \text { the observed process } X^{T} \text { is } \operatorname{TOU}(\vartheta), \vartheta \in \Theta
$$

Let us introduce the statistics

$$
\mathbb{W}_{T}^{2}=\frac{1}{\sigma^{2} T^{2}} \int_{0}^{T}\left[X_{t}-X_{0}-\int_{0}^{t} g\left(X_{s}, \vartheta_{T}^{*}\right) \mathrm{d} s\right]^{2} \mathrm{~d} t
$$

and

$$
\mathbb{D}_{T}=\frac{1}{\sigma \sqrt{T}} \sup _{0 \leq t \leq T}\left|X_{t}-X_{0}-\int_{0}^{t} g\left(X_{s}, \vartheta_{T}^{*}\right) \mathrm{d} s\right|,
$$

where $\vartheta_{T}^{*}$ is the maximum likelihood or bayesian estimator. Remind, that $\vartheta_{T}^{*}=\vartheta+\frac{u_{T}^{*}}{T}$. Using this singular rate of convergence of estimator it can be shown that under $\mathscr{H}_{0}$ we have the same limit distributions of the statistics

$$
\mathbb{W}_{T}^{2} \Longrightarrow \int_{0}^{1} W(s)^{2} \mathrm{~d} s, \quad \mathbb{D}_{T} \Longrightarrow \sup _{0 \leq s \leq 1}|W(s)|
$$

Hence the tests $\psi_{T}\left(X^{T}\right)=\mathbb{I}_{\left\{\mathbb{W}_{T}^{2}>c_{\alpha}\right\}}$ and $\phi_{T}\left(X^{T}\right)=\mathbb{I}_{\left\{\mathbb{D}_{T}>d_{\alpha}\right\}}$ are asymptotically distribution free. These tests as well are consistent against any fixed alternative.

The similar limits we have in the case of general model (15). For example, suppose that $\boldsymbol{\vartheta}_{0}$ is known and denote the trend coefficient in (15) as $S\left(\boldsymbol{\vartheta}_{0}, X_{t}\right)$. Then once more (under hypothesis)

$$
\mathbb{W}_{T}^{2}=\frac{1}{T^{2}} \int_{0}^{T}\left(\int_{0}^{t} \frac{\left[\mathrm{d} X_{s}-S\left(\boldsymbol{\vartheta}_{0}, X_{s}\right) \mathrm{d} s\right]}{\sigma\left(X_{s}\right)}\right)^{2} \mathrm{~d} t=\int_{0}^{1} W(s)^{2} \mathrm{~d} s .
$$

If the basic hypothesis is composite then the same statistic with $\boldsymbol{\vartheta}_{0}$ replaced by one of the estimators (MLE or BE) has this last integral as limit (in distribution).

It is interesting to study the direct analogs of the classical C-vM and $\mathrm{K}-\mathrm{S}$ tests. Let us introduce the empirical distribution function and empirical density (local time estimator)

$$
\hat{F}_{T}(x)=\frac{1}{T} \int_{0}^{T} \mathbb{I}_{\left\{X_{t}<x\right\}} \mathrm{d} t, \quad f_{T}^{\circ}(x)=\frac{\Lambda_{T}(x)}{T \sigma(x)^{2}} .
$$


Then the corresponding C-vM statistics

$$
\begin{aligned}
\mathbb{W}_{T}^{2} & =T \int_{-\infty}^{\infty}\left[\hat{F}_{T}(x)-F\left(\boldsymbol{\vartheta}_{0}, x\right)\right]^{2} \mathrm{~d} F\left(\boldsymbol{\vartheta}_{0}, x\right), \\
\mathbb{V}_{T}^{2} & =T \int_{-\infty}^{\infty}\left[f_{T}^{\circ}(x)-f\left(\boldsymbol{\vartheta}_{0}, x\right)\right]^{2} \mathrm{~d} F\left(\boldsymbol{\vartheta}_{0}, x\right)
\end{aligned}
$$

have limits in distribution but these limits are not distribution free [13]. One way to have asymptotically distribution free statistic was proposed by Negri and Nishiyama [17]. Another possibility (discussed in [14]) is to use the weight functions. Let us illustrate the second approach on the statistic

$$
\mathbb{V}_{T}^{2}\left(\boldsymbol{\vartheta}_{0}\right)=T \int_{-\infty}^{\infty} H\left(\boldsymbol{\vartheta}_{0}, x\right)\left(\hat{F}_{T}(x)-F\left(\boldsymbol{\vartheta}_{0}, x\right)\right)^{2} \mathrm{~d} F\left(\boldsymbol{\vartheta}_{0}, x\right)
$$

with weight function

$$
H\left(\boldsymbol{\vartheta}_{0}, x\right)=\frac{\Psi^{\prime}\left(\boldsymbol{\vartheta}_{0}, x\right)}{f\left(\boldsymbol{\vartheta}_{0}, x\right)\left[F\left(\boldsymbol{\vartheta}_{0}, x\right)-1\right]^{2}} M\left(\Psi\left(\boldsymbol{\vartheta}_{0}, x\right)\right) .
$$

where $M(\cdot)$ is some function providing the finitness of this integral and

$$
\begin{aligned}
\Psi\left(\boldsymbol{\vartheta}_{0}, x\right)=\int_{-\infty}^{x} \frac{F\left(\boldsymbol{\vartheta}_{0}, y\right)^{2}}{\sigma(y)^{2} f_{0}\left(\boldsymbol{\vartheta}_{0}, y\right)} \mathrm{d} y \\
\quad+F\left(\boldsymbol{\vartheta}_{0}, x\right)^{2} \int_{x}^{\infty}\left(\frac{F\left(\boldsymbol{\vartheta}_{0}, y\right)-1}{F\left(\boldsymbol{\vartheta}_{0}, x\right)-1}\right)^{2} \frac{\mathrm{d} y}{\sigma(y)^{2} f\left(\boldsymbol{\vartheta}_{0}, y\right)} .
\end{aligned}
$$

It is shown that if $M(s)=e^{-s}$ then

$$
\mathbb{V}_{T}^{2}\left(\boldsymbol{\vartheta}_{0}\right) \Longrightarrow \int_{0}^{\infty} W(s)^{2} e^{-s} \mathrm{~d} s
$$

where $W(\cdot)$ is a Wiener process, i.e.; we have asymptotically distribution free test $\hat{\psi}_{T}=\mathbb{I}_{\left\{\mathbb{v}_{T}^{2}\left(\boldsymbol{\vartheta}_{0}\right)>r_{\alpha}\right\}}$ [14]. The threshold $r_{\alpha}$, of course, is solution of the following equation

$$
\mathbf{P}\left\{\int_{0}^{\infty} W(s)^{2} e^{-s} \mathrm{~d} s>r_{\alpha}\right\}=\alpha .
$$

The similar result can be proved for the large class of functions $M(\cdot)$ satisfying the obvious conditions.

In the case of composite hypothesis we can replace $\boldsymbol{\vartheta}_{0}$ by one of the estimators, say, to use $\mathbb{V}_{T}^{2}\left(\widehat{\boldsymbol{\vartheta}}_{T}\right)$ and to have the same (distribution free) limit of this statistic. 


\section{References}

[1] Chan, K.S. (1993). Consistency and limiting distribution of the LSE of a TAR, Ann. Stat. 21, 520-533.

[2] Chan, N.H. and Kutoyants Yu.A. (2008) On parameter estimation of threshold autoregressive models, submitted.

[3] Dachian, S. and Kutoyants, Yu.A. (2007) On the goodness-of-fit tests for some continuous time processes, in Statistical Models and Methods for Biomedical and Technical Systems, F.Vonta et al. (Eds), Birkhäuser, Boston, 395-413.

[4] Durret, R. ( 1996) Stochastic Calculus: A Practical Introduction. Boca Raton: CRC Press.

[5] Fan, J. and Yao, Q. (2003). Nonlinear Time Series: Nonparametric and Parametric Methods. Springer, New York.

[6] Hansen, B.E. (2000). Sample splitting and threshold estimation, Econometrica 68, 575-603.

[7] Ibragimov, I.A. and Khasminskii, R.Z. (1981). Statistical Estimation. Springer, New York.

[8] Rubin, H. and Song, K.-S. (1995). Exact computation of the asymptotic efficiency of maximum likelihood estimators of a discontinuous signal in a Gaussian white noise. Ann. Stat.23, 732-739.

[9] Terent'yev, A.S. (1968). Probability distribution of a time location of an absolute maximum at the output of a synchronized filter, Radioengineering and Electronics, 13, 4, 652-657.

[10] Tong, H. (1990). Non-linear Time Series: A Dynamical Systems Approach. Oxford University Press, Oxford.

[11] Koul, H.L., Qianb, L. and Surgailis, D. (2003) Asymptotics of Mestimators in two-phase linear regression models. Stochastic. Process. Appl., 103, 123-154.

[12] Küchler, U., Kutoyants, Yu. A. (2000) Delay estimation for some stationary diffusion-type processes. Scand. J. Statist., 27, 3, 405-414.

[13] Kutoyants, Yu.A. (2004) Statistical Inference for Ergodic Diffusion Processes, Springer, London. 
[14] Kutoyants, Yu.A. (2008) On the goodness-of-fit testing for ergodic diffusion processes, to appear in Journal of Nonparametric Statistics.

[15] Lehmann, E.L. and Romano, J.P. (2005) Testing Statistical Hypotheses. (3rd ed.) Springer, N.Y.

[16] Liptser, R.S. and Shiryayev, A.N. (2001) Statistics of Random Processes. I, (2nd ed.) Springer, N.Y.

[17] Negri, I. and Nishiyama, Y. (2009) Goodness of fit test for ergodic diffusion processes. Annals of the Institute of Statistical Mathematics, 61,4, 919-928.

[18] Quandt, R.E., (1958) The estimation of the parameters of a linear regression system obeying two separate regimes. J. Amer. Statist. Assoc. $53,873-880$.

[19] Revuz, D. and Yor, M. (1991) Continuous Martingales and Brownian Motion. Springer, N.Y.

[20] Shreve, S.E. (2004) Stochastic Calculus for Finance II: Continuous-Time Models. Springer, N.Y.

[21] Yoshida, N.(2009) Polynomial type large deviation inequality and its applications. To appear in Annals of the Institute of Statistical Mathematics. 